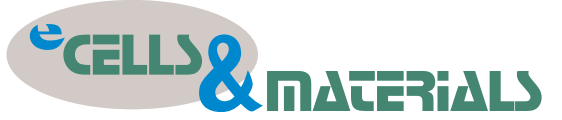

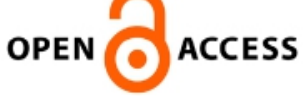

\section{MESENCHYMAL STEM CELL-MEDIATED ENDOCHONDRAL OSSIFICATION UTILISING MICROPELLETS AND BRIEF CHONDROGENIC PRIMING}

\author{
C.A. Knuth ${ }^{1}$, J. Witte-Bouma ${ }^{1}$, Y. Ridwan ${ }^{2}$, E.B. Wolvius ${ }^{1}$ and E. Farrell ${ }^{1}$ \\ ${ }^{1}$ Department of Oral and Maxillofacial Surgery, Special Dental Care and Orthodontics, Erasmus MC, \\ University Medical Centre, 3000 DR Rotterdam, The Netherlands \\ ${ }^{2}$ Department of Genetics, Cancer Genomics Centre, Erasmus MC, 3000 DR Rotterdam, The Netherlands
}

\begin{abstract}
With limited autologous and donor bone graft availability, there is an increasing need for alternative graft substitutes. We have previously shown that chondrogenically priming mesenchymal stem cell (MSC) pellets for $28 \mathrm{~d}$ in vitro will reproducibly result in endochondral bone formation after in vivo implantation. However, pellet priming time for clinical applications is quite extensive. A micropellet ( $\mu$ pellet)-fibrin construct was developed and coupled, with a shorter priming period, determined by an in vitro time course experiment. In vitro data showed expression of chondrogenic genes and matrix production after $7 \mathrm{~d}$ of chondrogenic priming, indicating that briefer priming could possibly be used to induce bone formation in vivo. 7 and $28 \mathrm{~d}$ primed pellet, pellet-fibrin and $\mu$ pellet-fibrin constructs were cultured for in vitro analysis and implanted subcutaneously for 8 weeks into nude mice. $\mu$ pellet-fibrin constructs, cultured in vitro for 7 or $28 \mathrm{~d}$, produced comparable bone to standard pellets in vivo. MSC-mediated bone formation was achieved following only $7 \mathrm{~d}$ of in vitro priming. Bone formation in vivo appeared to be influenced by overall matrix production pre-implantation. Given this short priming time and the injectable nature of the $\mu$ pellet-fibrin constructs, this approach might be further developed as an injectable bone substitute, leading to a minimally-invasive treatment option, which would allow for tailored filling of bone defects.
\end{abstract}

Keywords: Bone tissue engineering, endochondral ossification, fibrin, mesenchymal stem cells, hydrogel, bone, micropellet, chondrogenesis, marrow, injectable.

*Address for correspondence: Dr Eric Farrell, Department of Oral and Maxillofacial Surgery, Special Dental Care and Orthodontics, Erasmus MC, 3000 DR Rotterdam, The Netherlands.

Telephone: +31 107043655 Fax: +31 107033098 Email: e.farrell@erasmusmc.nl

\section{Introduction}

Availability of autologous graft material for reconstruction of large bone defects is limited and, as harvesting is associated with additional patient risk and discomfort, there is an increasing need for suitable graft alternatives (Amini et al., 2012; Damien and Parsons, 1991; Gawlitta et al., 2010).

Developmental engineering, mimicking the processes of tissue development and healing, to create biologically-relevant replacements for congenitally missing, worn out or damaged tissues, is commonly implemented in bone tissue engineering to develop replacements for autologous grafts (Badylak, 2005; Dimitriou et al., 2005). Bone formation predominantly proceeds through two mechanisms: intramembranous (IO) and endochondral ossification (EO). With varying degrees of success, both mechanisms are exploited for bone graft development, using stem cells and other cell types (Bahney et al., 2014; Huang et al., 2006; Olsen et al., 2000; Tortelli et al., 2010).
Although the IO route of bone formation is often used in tissue engineering (TE), grafts often exhibit poor survival due to insufficient vascularisation and perfusion following implantation (Sheehy et al., 2013). For these reasons, this work focused on achieving tissue engineered bone through the EO pathway.

Endochondral bone formation relies on a cartilage intermediate (Bianco et al., 1998; Maes et al., 2010). Mesenchymal cells condense and undergo chondrogenic differentiation, leading to the production of a cartilage template rich in collagens, glycosaminoglycans (GAGs) and bioactive signalling molecules (Kronenberg, 2003; Ortega et al., 2004). As the process continues, the chondrogenic matrix is calcified and degraded, resulting in the release of these molecules (Nagai and Aoki, 2002; Ortega et al., 2004; Street et al., 2002), which trigger cell migration, blood vessel invasion and ultimately bone formation (Boyan et al., 1992; Chen et al., 2012; Gawlitta et al., 2010; Gerber, 1999). It has previously been shown that EO can be mimicked in order to achieve bone formation 
using chondrogenically primed mesenchymal stem cell (MSC) pellets (Farrell et al., 2011; van der Stok et al., 2014). Chondrogenically differentiated MSC pellets in vitro for $28 \mathrm{~d}$ result in vascularised, endochondral bone when subcutaneously implanted in vivo for 8 weeks. This approach to TE bone formation is advantageous, as cartilage is better suited to survive in the initial avascular environment and naturally promotes the revascularisation of the graft material when implanted into defect sites (Yang et al., 2014). Whilst a promising approach to bone regeneration, hurdles remain when trying to implement such techniques clinically. A major obstacle is the lengthy in vitro priming time required to differentiate pellets to stimulate endochondral ossification in vivo, a process which is essential as undifferentiated MSC pellets typically fail to form bone or form an insufficient quantity to heal defects (van der Stok et al., 2014). It has recently been shown that in vitro priming can be reduced to $14 \mathrm{~d}$; however, it has not been investigated whether further reduction is possible, to allow for a swifter patient treatment (Yang et al., 2015). In vitro priming time is further compounded by the fact that inherent variation among MSC donors result in varying degrees of chondrogenic differentiation, which can affect the quantity and quality of bone produced in vivo (Osinga et al., 2016; Scotti et al., 2010; van der Stok et al., 2014). In order for these constructs to be clinically relevant, criteria for in vitro differentiation, which are shown to lead to reproducible bone formation, must be identified. However, in vitro priming and donor variation are not the only obstacles between these constructs and clinical translation. Isolation of MSCs from bone marrow requires a painful surgical procedure, always followed by a secondary surgical procedure - subsequent to the construction of the MSC pellets - required for the implantation of the graft. The development of an injectable bone substitute would allow for a less invasive treatment option and, possibly, decreased recovery time.

To address these issues, we have created chondrogenically primed constructs comprised of micropellets ( $\mu$ pellets) suspended within a fibrin hydrogel. This approach exploits the reproducible and swift bone-forming capacity of MSC pellets, coupled with a shorter in vitro priming time to decrease the pre-implantation time. $\mu$ pellet constructs are promising constructs since their small size allows them to pass easily through a hypodermic needle, making them ideal for an injectable therapy. This would allow clinicians to properly fill a defect site without the need for a tailor-printed/designed implant to fit specific void shapes and it would result in decreased patient discomfort and possibly recovery time.

In this study, we aimed to examine the boneforming potential of these $\mu$ pellet constructs. We investigated decreased in vitro priming with the goal of achieving bone formation in a shorter time than the one so far reported. Additionally, we observed how differences in chondrogenic potential preimplantation influence endochondral bone formed in vivo.

\section{Materials and Methods}

\section{MSC isolation}

Human bone marrow MSCs were isolated from 3 different consenting patients undergoing total hip arthroplasty [Medical Ethical Testing Commission (METC) approval code 2004-142; donor 1: female, 60 years old; donor 2: male, 34 years old; donor 3: male, 58 years old] and expanded in alpha modifications minimum essential medium ( $\alpha \mathrm{MEM})$ containing $10 \%$ foetal bovine serum (FBS) serum (lot \#41Q204K, Gibco, Bleiswijk, The Netherlands) and supplemented with $50 \mu \mathrm{g} / \mathrm{mL}$ gentamycin, $1.5 \mu \mathrm{g} /$ $\mathrm{mL}$ fungizone, $25 \mu \mathrm{g} / \mathrm{mL}$ L-ascorbic acid 2-phosphate and $1 \mathrm{ng} / \mathrm{mL}$ fibroblast growth factor-2 (all from Instruchemie, Delfzijl, The Netherlands). Heparinised bone marrow aspirates were taken from the greater trochanter and plated at the concentration of 30$100 \times 10^{6}$ nucleated cells per T175 flask (Corning, Amsterdam, The Netherlands). $24 \mathrm{~h}$ after plating, flasks were washed to remove non-adherent cells and debris and the medium was changed. Cells were cultured at $37^{\circ} \mathrm{C}$ and $5 \%$ carbon dioxide $\left(\mathrm{CO}_{2}\right)$ and medium was changed twice a week. MSCs were passed once reached 85-90\% confluence using $0.05 \%$ trypsin (Gibco) and replated at a concentration of approximately 2,800 cells $/ \mathrm{cm}^{2}$. Cells were expanded to passage-4, at which time they had differentiated chondrogenically.

\section{Chondrogenic differentiation}

To create standard pellets, $2 \times 10^{5}$ MSCs were suspended in $15 \mathrm{~mL}$ polypropylene tubes in complete chondrogenic medium [high-glucose Dulbecco's modified Eagle's medium (DMEM) supplemented with $50 \mu \mathrm{g} / \mathrm{mL}$ gentamycin (Invitrogen, Landsmeer, The Netherlands), $1.5 \mu \mathrm{g} / \mathrm{mL}$ fungizone (Invitrogen), $1 \mathrm{mM}$ sodium pyruvate (Invitrogen), $40 \mu \mathrm{g} / \mathrm{mL}$ proline (Sigma-Aldrich, Zwijndrecht, The Netherlands), $1: 100 \mathrm{v} / \mathrm{v}$ insulin-transferrin-selenium (ITS+; BD Biosciences, Temse, Beligum), $10 \mathrm{ng} / \mathrm{mL}$ transforming growth factor $\beta 3$ (Peprotech, London, UK), 25 mg/ $\mathrm{mL}$ L-ascorbic acid 2-phosphate (Sigma-Aldrich) and $100 \mathrm{nM}$ dexamethasone (Sigma-Aldrich)] and centrifuged for $8 \mathrm{~min}$ at $200 \times g$. To generate $\mu$ pellets, $3 \times 10^{6}$ MSC s per well were seeded in AggreWell ${ }^{\text {TM}} 800$ plates (STEMCELL Technologies, Evergem, Belgium) in $1 \mathrm{~mL}$ chondrogenic medium, centrifuged at $300 \times g$ for $10 \mathrm{~min}$ and incubated overnight creating 300 upellets (10,000 cells per $\mu$ pellet) per well. Following $24 \mathrm{~h}$ of incubation, $\mu$ pellets were transferred to 24 well plates (Corning, Zwijndrecht, The Netherlands) or fibrin encapsulated. Cells undergoing chondrogenic differentiation were cultured at a ratio of $4 \times 10^{5}$ cells/mL in complete chondrogenic medium. All conditions were cultured at $37^{\circ} \mathrm{C}$ in $5 \% \mathrm{CO}_{2}$. 


\section{Chondrogenic time course}

MSCs(donor information above) were chondrogenically differentiated through standard pellet culture conditions, as described. For histological analysis, samples were harvested following 2, 5, 7, 10, 14 and $28 \mathrm{~d}$ of differentiation. Samples were fixed for $2 \mathrm{~h}$ in $4 \%(\mathrm{w} / \mathrm{v})$ formaldehyde in PBS. Samples for biochemical assays were harvested after 7, 14, 21 and $28 \mathrm{~d}$, digested overnight at $60^{\circ} \mathrm{C}$ in buffer containing papain $\left[0.2 \mathrm{M} \mathrm{NaH}_{2} \mathrm{PO}_{4}, 0.01 \mathrm{M} \mathrm{EDTA}_{2} \mathrm{H}_{2} \mathrm{O}, 0.01 \mathrm{M}\right.$ cysteine $\mathrm{HCl}, 250 \mu \mathrm{g} / \mathrm{mL}$ papain (Sigma-Aldrich), $\mathrm{pH} 6$ ] and stored at $-20^{\circ} \mathrm{C}$ until use. Samples for gene expression analysis were harvested after 2, 5, 7, 10 and $14 \mathrm{~d}$ of chondrogenic differentiation, homogenised in $350 \mu \mathrm{L}$ of TRIzol (Gibco) and stored at $-80^{\circ} \mathrm{C}$ until use.

\section{Fibrin encapsulation}

Pellets and $\mu$ pellets were collected $24 \mathrm{~h}$ post-pelleting and suspended in $100 \mu \mathrm{L}$ of $40 \mathrm{mg} / \mathrm{mL}$ human fibrinogen, dissolved in $0.9 \% \mathrm{NaCl}$ (Sigma-Aldrich) at a density of 60 upellets or 3 standard pellets per $100 \mu \mathrm{L}$ of fibrinogen. Human thrombin (SigmaAldrich) was added at a ratio of 0.5 international units (IU) to $1 \mathrm{mg}$ of fibrinogen; crosslinking took 15-20 min. Samples for implantation and in vitro analysis were maintained for 7 or $28 \mathrm{~d}$ in complete chondrogenic medium supplemented with $0.0875 \mathrm{IU} /$ $\mathrm{mL}$ of bovine aprotinin (Sigma-Aldrich), to slowdown the degradation of the fibrin material caused by cell constructs during the in vitro culture. Medim was refreshed twice a week and pellets weremainteined at $37^{\circ} \mathrm{C}$ in $5 \% \mathrm{CO}_{2}$.

\section{Subcutaneous implantation}

All experiments were approved by the Animal Experiments Committee at the Erasmus Medical Centre, Rotterdam, The Netherlands (DEC protocol 116-12-08). 3 loose pellets, 60 loose $\mu$ pellets, 3 fibrinencapsulated pellets and 60 fibrin-encapsulated upellets (for a total of 600,000 cells per pocket) were implanted subcutaneously in 10 (7 d in vitro primed samples) or 13 weeks old ( $28 \mathrm{~d}$ in vitro primed samples) male athymic nude mice (Balb/c nudes, Charles River, Sulzfeld, Germany). Animal were housed in groups of three under a standard $12 \mathrm{~h}$ light/ dark cycle, with access to food and water ad libitum (18 mice in total). Pre-operatively, animals were given pain medication (buprenorphine, $0.05 \mathrm{mg} /$ $\mathrm{kg}$ body weight) under general anaesthesia (1-3.5\% isoflurane). Two incisions were made dorsally (one between the shoulder blades and the other between the hip bones), with two subcutaneous pockets created per incision. Implantation per pocket was performed according to one of the following conditions: 3 standard pellets, 3 standard pellets encapsulated in fibrin, 60 loose $\mu$ pellets or 60 ppellets encapsulated in fibrin (implantation was randomised among pockets and animals). 3 replicates per condition and MSC donor (3 MSC donors in total) were implanted. 8 weeks post implantation, animals were euthanised by $\mathrm{CO}_{2}$ asphyxiation and constructs were retrieved.

\section{Micro-computed tomography $(\mu \mathrm{CT})$ imaging}

$\mu \mathrm{CT}$ scans were performed and reconstructed at the Applied Molecular Imaging Erasmus MC facility using the Quantum FX imaging systems (PerkinElmer, Groningen, The Netherlands). Ex vivo scans were acquired using a field of view of $10 \mathrm{~mm}$ (90 kV/160 mA, 3 min). Scans were quantified using the Analyze 11.0 software (AnalyzeDirect, Nieuwe Niedorp, The Netherlands). Calcified tissue was quantified by converting original linear attenuation coefficient measurements, by linear transformation, to Hounsfield units (HU). Global thresholding was applied to all scans and determined by visual inspection. Values over $400 \mathrm{HU}$, corresponding to $0.133 \mathrm{~g} / \mathrm{cm}^{3}$ (as determined by phantom scans), were segmented out and independently quantified as calcified tissue.

\section{Histology}

Samples were fixed for $2 \mathrm{~h}$ (in vitro) or $24 \mathrm{~h}$ (in vivo) in $4 \%(\mathrm{w} / \mathrm{v})$ formaldehyde in PBS. In vivo samples were decalcified for 7 to $10 \mathrm{~d}$ in $10 \% \mathrm{w} / \mathrm{v}$ EDTA in PBS, changing the solution every other day, then embedded in paraffin wax. $6 \mu \mathrm{m}$-thick sections were cut, deparaffinised and stained with haematoxylin

Table 1. Primer sequences used for real-time PCR. Abbreviations: GAPDH, glyceraldehyde3-phosphate dehydrogenase; VEGFa, vascular endothelial growth factor a; COLI, collagen type I; COLII, collagen type II; COLX, collagen type X; BMP2, bone morphogenetic protein 2; MMP13, matrix metallopeptidase 13.

\begin{tabular}{|c|c|c|}
\hline $\begin{array}{c}\text { Primer } \\
\text { Name }\end{array}$ & Forward sequence $\mathbf{( 5}^{\prime} \mathbf{- 3}^{\prime} \mathbf{)}$ & Reverse sequence $\mathbf{( 5}^{\prime} \mathbf{- 3}^{\prime} \mathbf{)}$ \\
\hline GAPDH & ATGGGGAAGGTGAAGGTCG & TAAAAGCAGCCCTGGTGACC \\
\hline VEGFa & CTTGCCTTGCTGCTCTACC & CACACAGGATGGCTTGAAG \\
\hline COLI & CAGCCGCTTCACCTACAGC & TTTTGTATTCAATCACTGTCTTGCC \\
\hline COLII & CCCCATCTGCCCAACTGA & CTCCTTTCTGTCCCTTTGGT \\
\hline COLX & ACTTCTCTTACCACATACACG & CCAGGTAGCCCTTGATGATGTACT \\
\hline BMP2 & AACACTGTGCGCAGCTTCC & CTCCGGGTTGTTTTCCCAC \\
\hline MMP13 & AAGGAGCATGGCGACTTCT & TGGCCCAGGAGGAAAAGC \\
\hline
\end{tabular}


and eosin (H\&E), safranin $\mathrm{O}$ or tartrate-resistant acid phosphatase (TRAP). 4-5 sections per sample were used. H\&E staining was performed by incubating deparaffinised samples for $5 \mathrm{~min}$ in $100 \%$ Gil's haematoxylin (Sigma-Aldrich), followed by $7 \mathrm{~min}$ incubation in non-distilled water. Next, samples were incubated for $45 \mathrm{~s}$ in $2 \%$ eosin (Merck, Amsterdam, The Netherlands) in $50 \%$ ethanol and $0.5 \%$ acetic acid for $10 \mathrm{~s}$ in $70 \%$ ethanol, followed by $100 \%$ ethanol for $1 \mathrm{~min}$ and $100 \%$ xylene for $1 \mathrm{~min}$ and mounted on coverslips. H\&E stained sections were used to quantify the percentage of bone, calcified cartilage and bone marrow - using morphological characteristics to determine boundaries. Aforementioned areas were segmented and then quantified using Image J software (National Institute of Health; Bethesda, MD, USA). Safranin O staining was performed by incubating deparaffinised samples for $8 \mathrm{~min}$ in $0.05 \%$ light green solution (Sigma-Aldrich) in distilled water, followed by a rinse in $1 \%$ acetic acid (Fluka, Zwijndrecht, The Netherlands) in distilled water and a $12 \mathrm{~min}$ incubation in $0.1 \%$ safranin $\mathrm{O}$ solution (Fluka) in distilled water. Subsequently, samples were rinsed for $30 \mathrm{~s}$ in $96 \%$ ethanol, followed by $100 \%$ ethanol for $1 \mathrm{~min}$ and $100 \%$ xylene for $1 \mathrm{~min}$ and mounted on coverslips. TRAP staining was performed by incubating deparaffinised samples for $20 \mathrm{~min}$ in $0.2 \mathrm{M}$ acetate buffer [0.2 M sodium acetate (Sigma-Aldrich), 100 nM L-(+)-tartaric acid (Acros, Antwerp, Beligum) pH 5]. Following incubation, $0.5 \mathrm{mg} / \mathrm{mL}$ naphtol AS-BI phosphate (Sigma-Aldrich) and $1.1 \mathrm{mg} / \mathrm{mL}$ fast red TR salt (Sigma-Aldrich) were added to the acetate buffer and incubated for $1 \mathrm{~h}$ at $37^{\circ} \mathrm{C}$. Collagen type II samples were rinsed in distilled water and counterstained with haematoxylin, as previously described.

\section{Immunohistochemistry}

Samples were prepared as previously described (Hellingman et al., 2010). Briefly, collagen type IIstained samples were incubated for $30 \mathrm{~min}$ at $37^{\circ} \mathrm{C}$ in $1 \mathrm{mg} / \mathrm{mL}$ pronase (Sigma-Aldrich) and $10 \mathrm{mg} /$ $\mathrm{mL}$ hyaluronidase (Sigma-Aldrich). $10 \%$ normal goat serum (Southern Biotech, Uithoorn, The Netherlands) was used to block non-specific antibody binding. Sections were incubated with $0.4 \mathrm{mg} / \mathrm{mL}$ of mouse anti-collagen type II antibody (II-II/II6B3; Developmental Studies Hybridoma Bank, University of Iowa, Iowa City, IA, USA). Collagen type X staining was performed by incubating samples with $0.1 \%$ pepsin (Sigma-Aldrich) in $0.5 \mathrm{M}$ acetic acid ( $\mathrm{pH}$ 2.0), followed by $10 \mathrm{mg} / \mathrm{mL}$ hyaluronidase treatment. Rat knees were decalcified for 2 weeks in $10 \%$ EDTA (w/v in distilled water; Sigma-Aldrich). Sections were incubated for $16 \mathrm{~h}$ with $1: 10$ or $1: 100$ diluted mouse anti-collagen type X antibody (X53; Quartett, Berlin, Germany; in PBS/1 \% BSA). All stainings were incubated for $30 \mathrm{~min}$ with $2.2 \mathrm{mg} / \mathrm{mL}$ of biotinSP-AffinityPure F(ab)2 goat anti-mouse antibody (Jackson Huissen, The Netherlands) diluted in PBS/1 \% BSA and washed in PBS. Next, samples were incubated for 30 min with streptavidin-AP (Biogenex, Uithoorn, The Netherlands) diluted 1:50 in PBS/1 \% BSA and washed in PBS. All slides were incubated for $30 \mathrm{~min}$ in $0.2 \mathrm{M}$ Tris-HCL substrate ( $\mathrm{pH} 8.5)$, containing $1 \mathrm{~g} / 25 \mathrm{~mL}$ Neu Fuchsin (1B467; Fisher Scientific, Landsmeer, The Netherlands) in $2 \mathrm{M} \mathrm{HCl}$, $4 \% \mathrm{NaNO}_{2}$ (Sigma-Aldrich) in distilled water, $0.3 \mathrm{mg} /$

a.

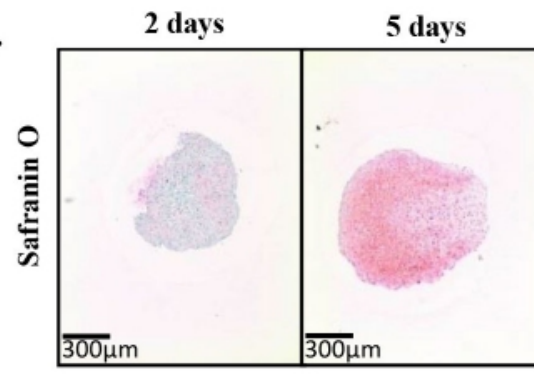

7 days

10 days

14 days

28 days
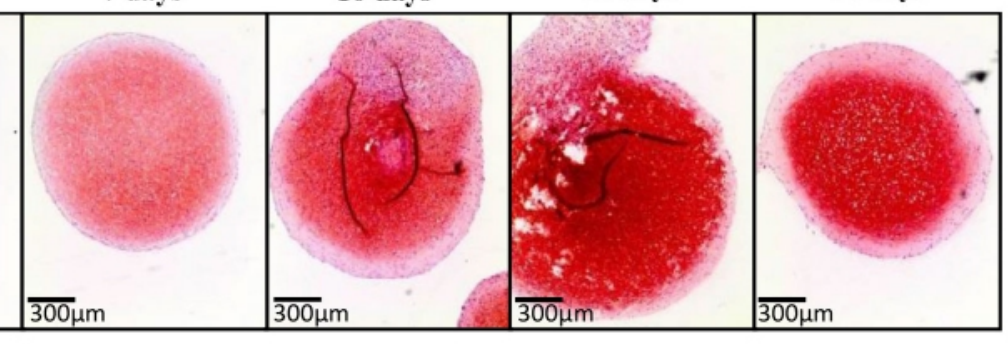

b.

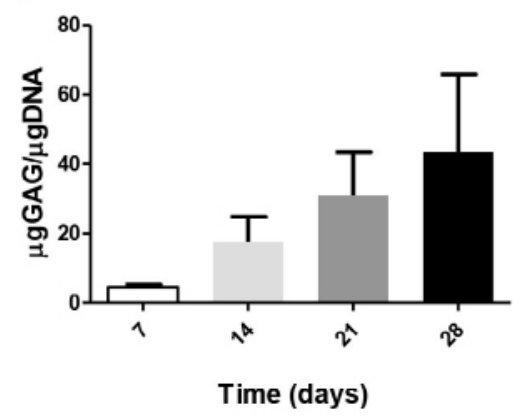

c.

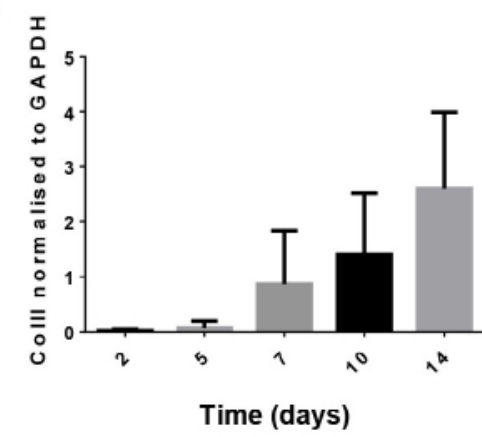

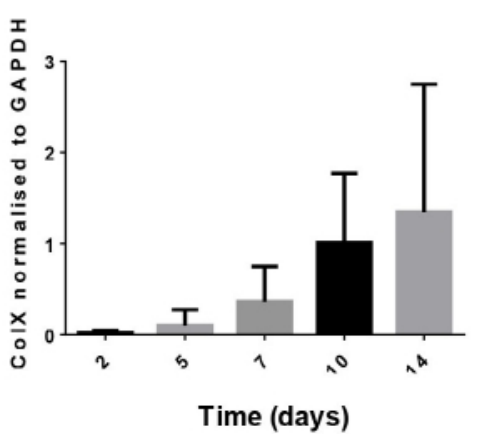

Fig. 1. Chondrogenic markers were upregulated after $7 \mathrm{~d}$ of in vitro priming. (a) Safranin $\mathrm{O}$ staining (donor 1) and (b) GAG (corrected for DNA) results showed an increase in matrix GAG deposition after $7 \mathrm{~d}$ of culture (all 3 MSC donors, 3 pellets per donor). (c) Expression of ColII and ColX increased after $7 \mathrm{~d}$ of in vitro culture and continued to increase over time (all $3 \mathrm{MSC}$ donors, 3 pellets per donor; graphs display mean and standard deviation). 
mL Naphtol AS-MX phosphate (Sigma-Aldrich), $33 \mu \mathrm{L} / \mathrm{mL}$ dimethylformamide (Sigma-Aldrich) and $0.25 \mathrm{mg} / \mathrm{mL}$ levamisole (Sigma-Aldrich). Collagen type II samples were rinsed in PBS, followed by haematoxylin counterstaining. Matching mouse IgG (X0931; Dako, Fisher Scientific) isotype controls were performed for each staining.

\section{Biochemical assays}

Samples were digested overnight at $60{ }^{\circ} \mathrm{C}$ in buffer containing papain (as described above). Glycosaminoglycan (GAG) content was quantified by dimethylmethylene blue (DMB) pH3 (Sigma-Aldrich) assay. Chondroitin sulphate C (Sigma-Aldrich) was used to create a standard for this experiment and a.
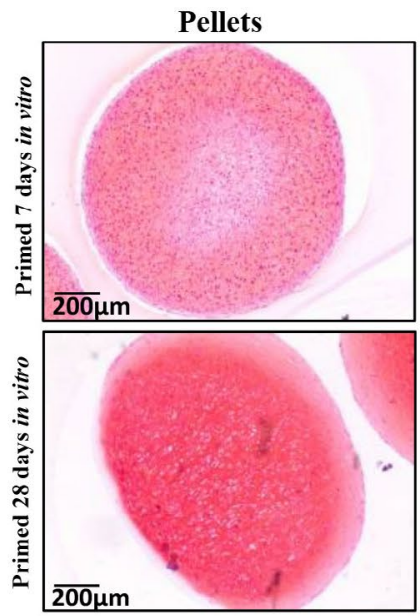

b.

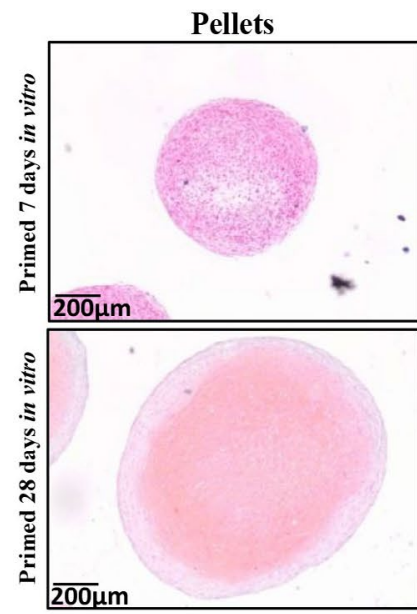

c.
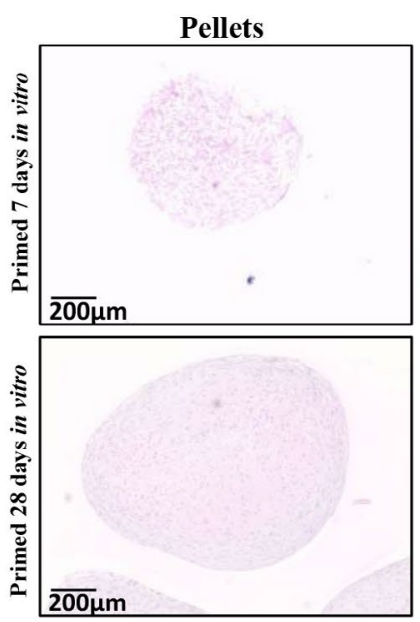

Pellets in fibrin
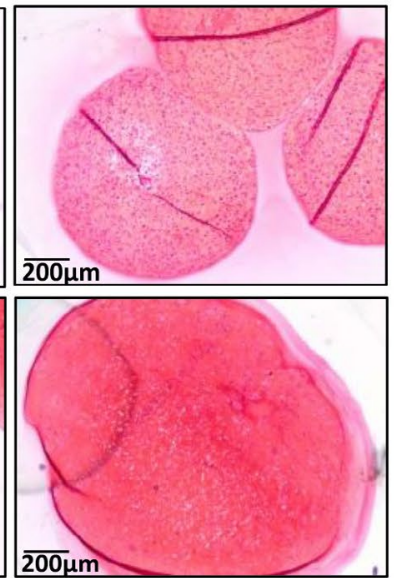

Pellets in fibrin

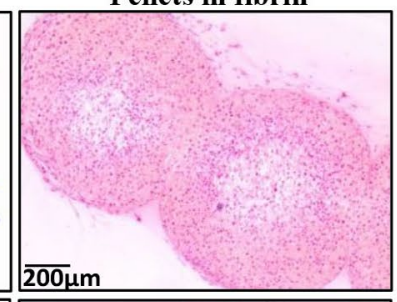

$\overline{200 \mu \mathrm{m}}$

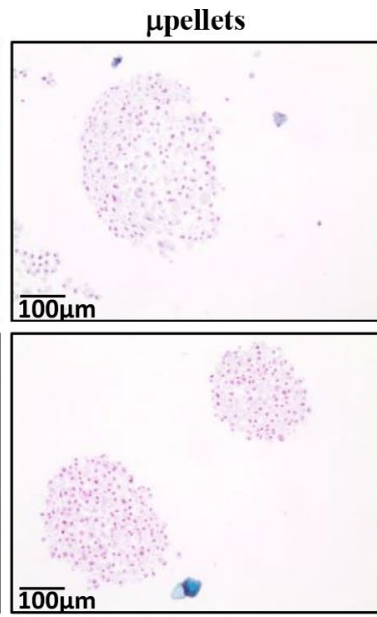

upellets
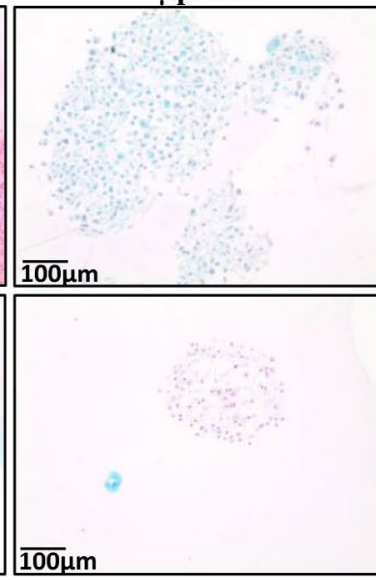

$\overline{200 \mu m}$

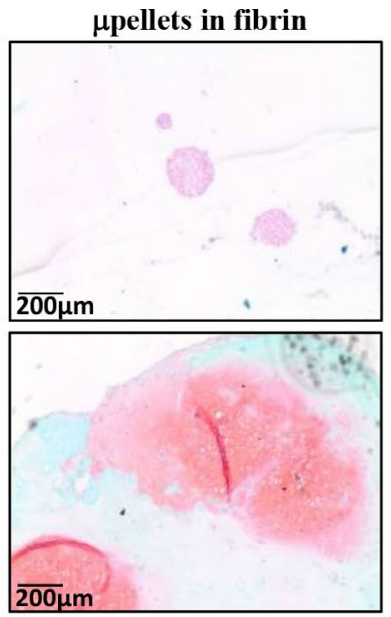

$\mu$ pellets in fibrin

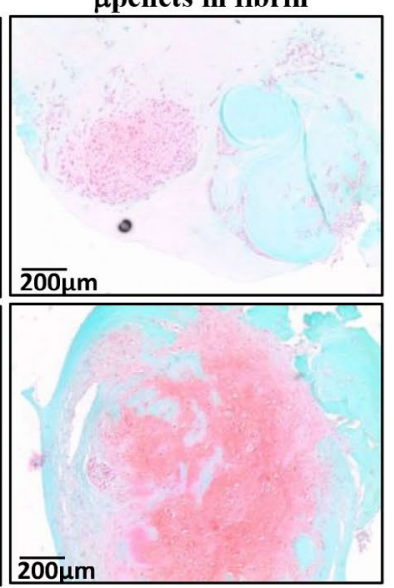

$\mu$ pellets in fibrin

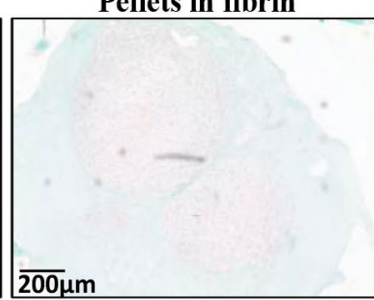

$\overline{100} \mu \mathrm{m}$

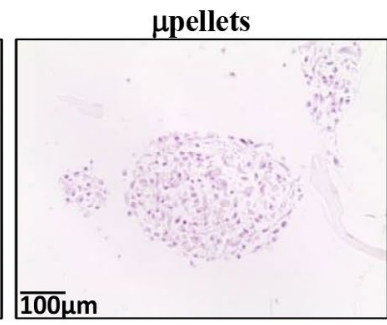

$\overline{200 \mu \mathrm{m}}$
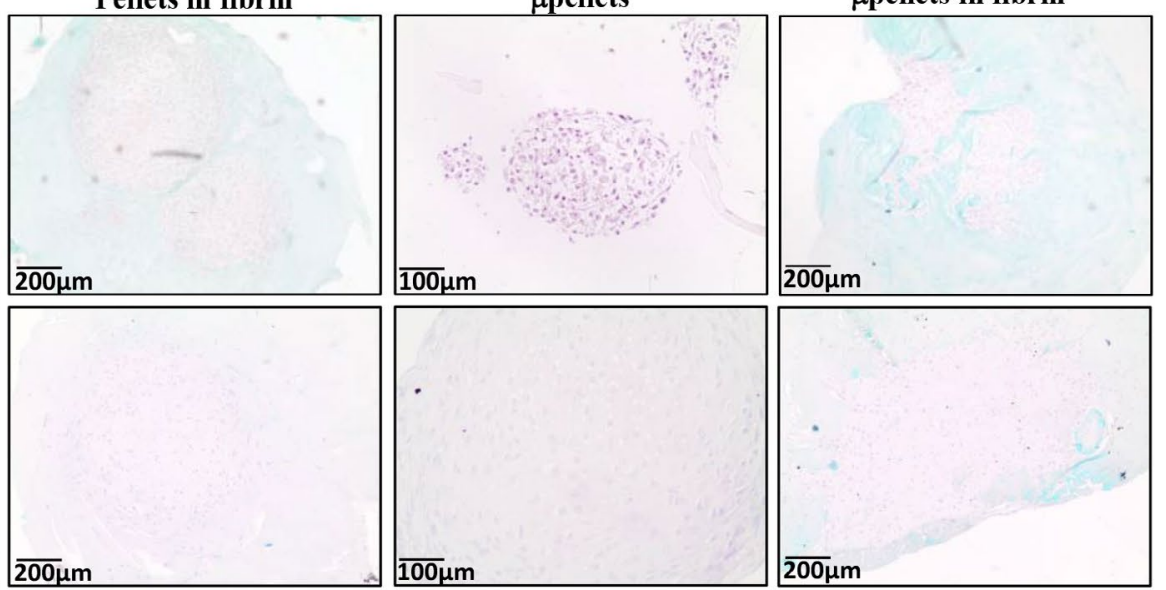

Fig. 2. Non-encapsulated $\mu$ pellets failed to differentiate chondrogenically. Safranin O results showed that non-encapsulated $\mu$ pellets exhibited no matrix GAG deposition after 7 or $28 \mathrm{~d}$ of culture [(a) donors 1 and (b) donor 2], whereas other constructs showed positive staining. (c) Donor $3 \mu$ pellets aggregated forming one pellet after $28 \mathrm{~d}$ in culture. 
the absorbance ratio $530: 590$ was used to determine the GAG content. DNA was quantified from papain digested samples using ethidium bromide with calf thymus DNA (Sigma-Aldrich) as a standard.

\section{Gene expression}

Samples were homogenised in $350 \mu \mathrm{L}$ of TRIzol (Gibco). $70 \mu \mathrm{L}$ of $100 \%$ chloroform were added and samples were agitated, incubated for $10 \mathrm{~min}$ at room temperature and then the aqueous phase was transferred to RNeasy ${ }^{\circledR}$ micro kit columns (Qiagen, Venlo, The Netherlands) for RNA extraction. cDNA was reverse transcribed using a First Strand cDNA Synthesis Kit (RevertAid, MBI Fermentas, St. Leon-Rot, Germany), according to manufactures instructions. Real-time PCR was performed using $10 \mathrm{ng}$ of cDNA. Samples were amplified for a maximum of 40 cycles using either SYBR Green I dye (Eurogentec, Seraing, Belgium) or TaqMan $2 \times$ Reagent (Applied Biosystems, Nieuwerkerk a/d, Ijssel, The Netherlands) in $10 \mu \mathrm{L}$ PCR mix reactions containing $10 \mu \mathrm{M}$ forward and reverse primers for GAPDH, COLII, COLX, COLI, VEGF, MMP13 or BMP2 (Table 1). For gene expression analysis, 3 different MSC donors were analysed with three pellets per donor. Gene expression was calculated by the $2-\Delta \Delta \mathrm{Ct}$ method.

\section{Results}

\section{MSCs displayed chondrogenic characteristics} during the first week of in vitro priming

The effects of in vitro chondrogenic priming time were investigated in order to determine the earliest stable chondrogenic induction time that could be utilised for in vivo bone formation studies. Time course experiments, in which MSC pellets were differentiated for $2,5,7,10,14,21$ or $28 \mathrm{~d}$ showed that GAGs were present early during priming and increased greatly by $7 \mathrm{~d}$ of in vitro culture, compared to earlier time points (Fig. 1a,b). Increased expression of chondrogenic genes, including COLII and COLX, was observed by $7 \mathrm{~d}$ and continued to rise thereafter compared to time point 0 (Fig.1c). As chondrogenic characteristics were observed to be stably and reproducibly increased after $7 \mathrm{~d}$ of in vitro priming, compared to earlier time points, we chose to continue with this time point during in vivo studies.

\section{upellet-fibrin constructs maintained chondrogenic potential compared to standard pellets}

$\mu$ pellets were constructed using roughly 10,000 cells per $\mu$ pellet, 20 of these $\mu$ pellets were equivalent to the cell density of our standard pellet, which is typically utilised in tissue engineering EO studies. upellets were encapsulated in a $40 \mathrm{mg} / \mathrm{mL}$ fibrin gel. To ensure fibrin was not inhibiting chondrogenesis, the pellets were also encapsulated for comparison. Pellets, pellet-fibrin, $\mu$ pellets and $\mu$ pellet-fibrin were cultured for either 7 or $28 \mathrm{~d}$ and then harvested for in vitro analysis or subcutaneously implanted in nude mice. In vitro, upellets alone failed to differentiate chondrogenically after 7 or $28 \mathrm{~d}$, fibrin encapsulation was necessary in order to achieve differentiation (Fig. 2). In donor 3, upellets aggregated following prolonged culture $(28 \mathrm{~d})$, forming one pellet with a decreased chondrogenic potential compared to standard pellets cultured from the same donor (Fig 2c). Safranin O staining suggested a similarity in proteoglycan content among pellet, pellet-fibrin and upellet-fibrin constructs after either 7 or $28 \mathrm{~d}$ of priming, as staining intensity was comparable between conditions. Although in earlier primed samples it might appear as if the staining of $\mu$ pelletfibrin condition was less intense, the pellets were spread over a larger area, distributing the staining over the section and the cumulative result was similar to that observed in the standard pellet culture. Among donors, there was variation in staining intensity highlighting a degree of donor variability during a.

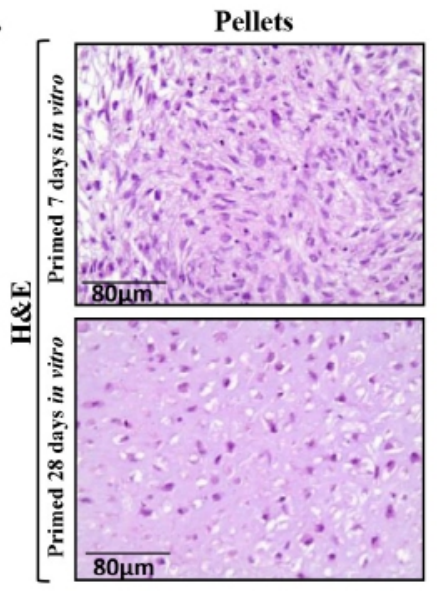

Pellets in fibrin

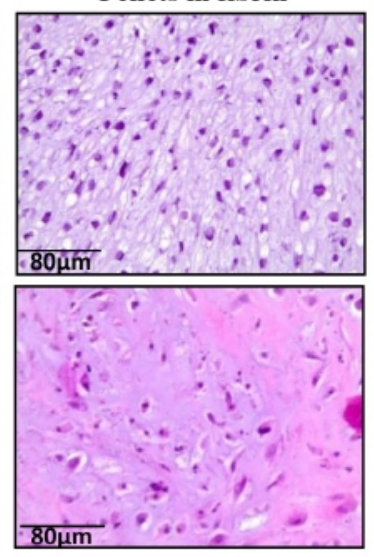

$\mu$ pellets in fibrin

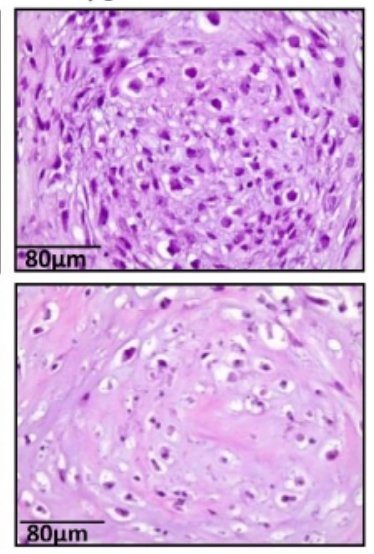

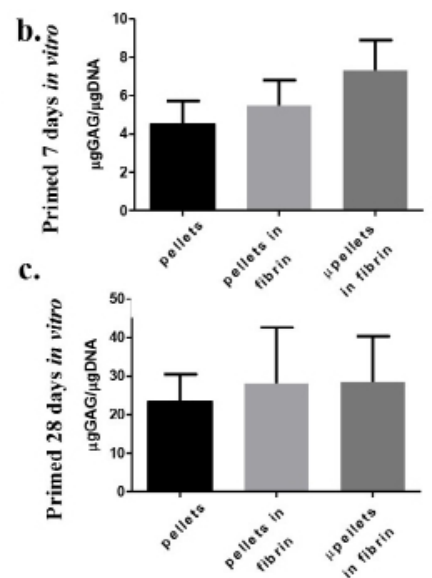

Fig. 3. upellet-fibrin constructs maintained cell morphology and exhibited chondrogenic potential. H\&E staining showing morphology of chondrogenic pellet, pellet-fibrin and upellet-fibrin (donor 2) was comparable among conditions, after 7 and $28 \mathrm{~d}$ of differentiation. 
chondrogenesis (Fig. 2). Cell morphology for each donor was similar among conditions and time points (Fig. 3a). However, after $7 \mathrm{~d}$ of culture, GAG/DNA data for donor 2 showed that $\mu$ pellet-fibrin constructs contained more GAG compared to pellet counterparts (Fig 3b; one donor, 6 replicates). This difference was not observed after extended culture (Fig. 3c; one donor, 6 replicates).

Expression of COLII, COLX and COLI was observed in all donors after either 7 or $28 \mathrm{~d}$ of in vitro culture; however, gene expression levels varied

a.

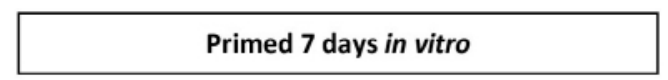

b.
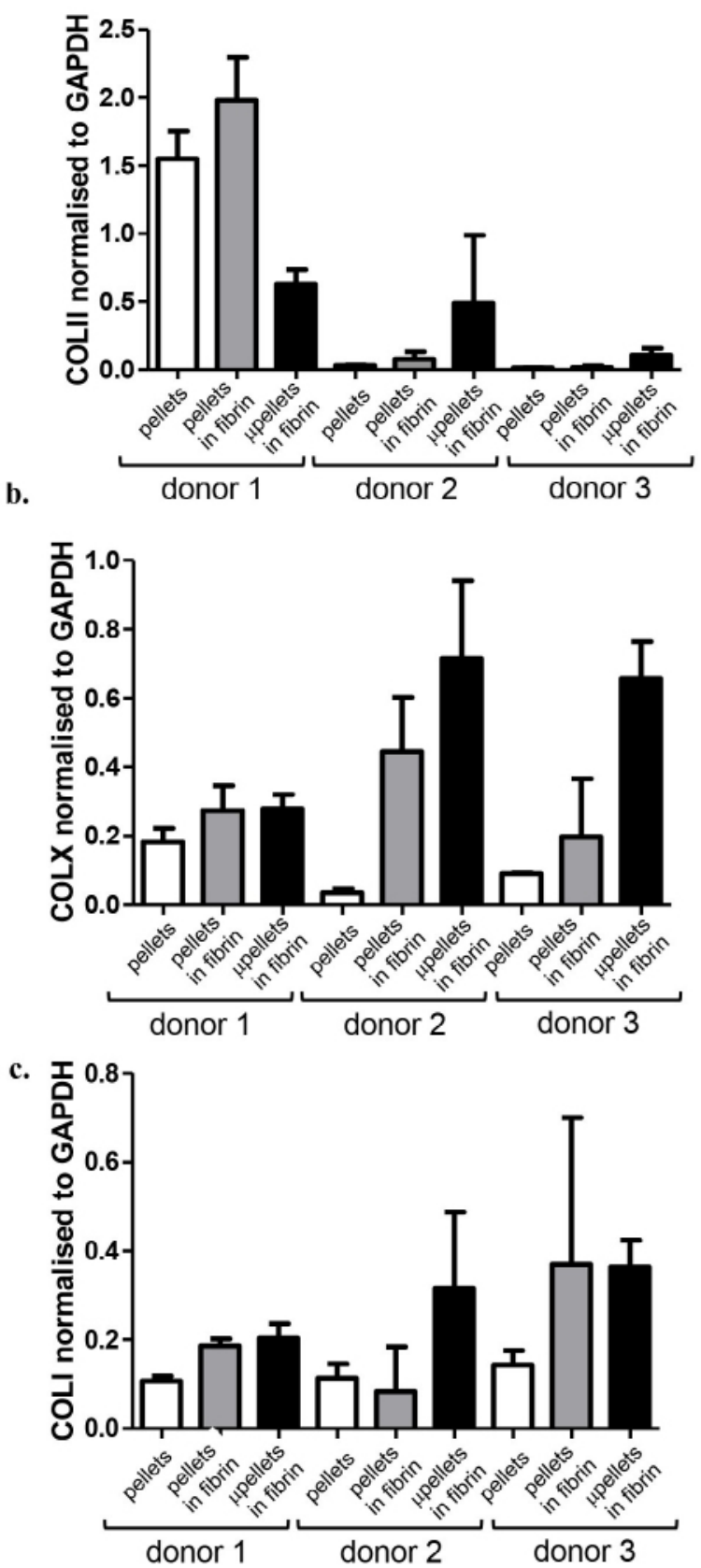

significantly among donors (Fig. 4). Samples collected from donor 1 , after both 7 and $28 \mathrm{~d}$ of culture, showed stronger expression and staining of COLII (Fig. $4 \mathbf{a}, 5)$. COLX expression was greater in $\mu$ pellet-fibrin compared to pellets alone, at both 7 and $28 \mathrm{~d}$ (Fig. 4 b). Fibrin encapsulation appeared to lead to a more intense COLII staining throughout the matrix after 7 or $28 \mathrm{~d}$ of culture, as shown by immunohistochemical analysis (Fig. 5). There was no clear variation in COLX protein expression among conditions (Fig. 6). In IgG control stainings, COLX appeared to be slightly
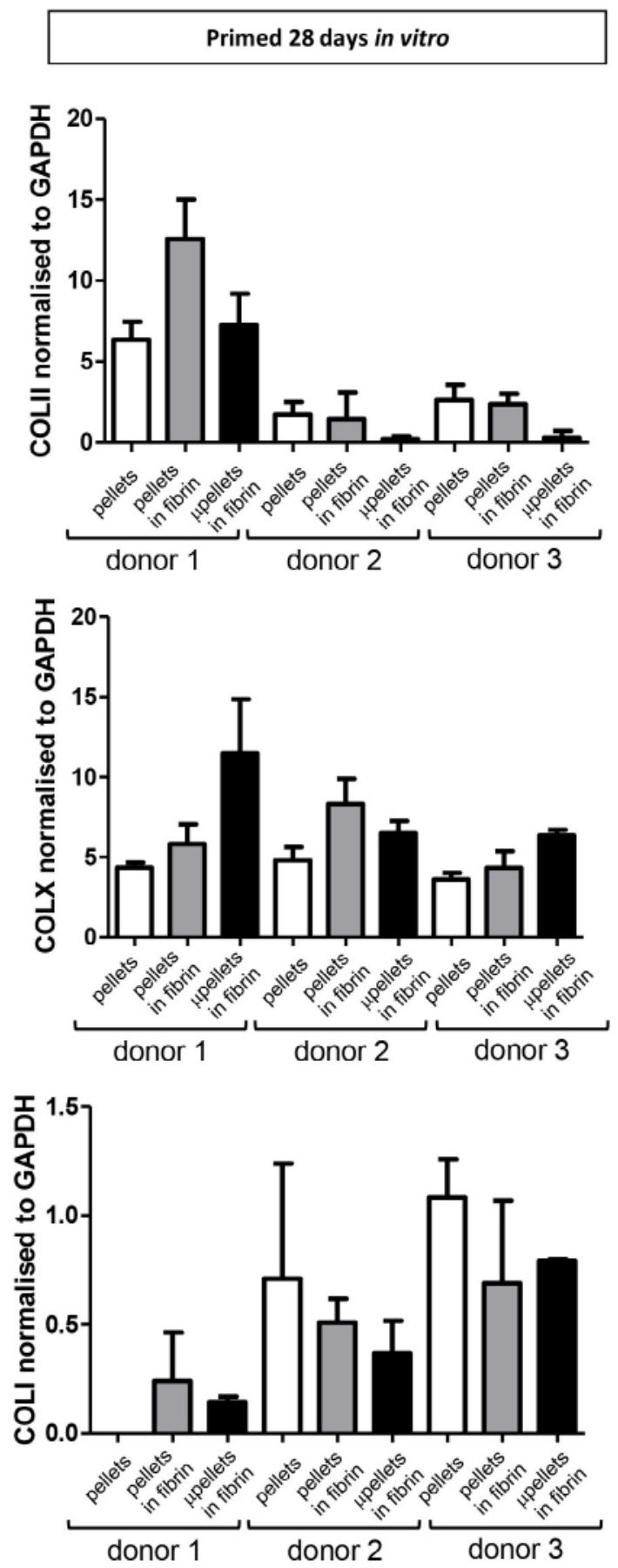

Fig. 4. Matrix components were differentially affected among donors; however, hypertrophic marker COLX was consistently upregulated in $\mu$ pellet-fibrin constructs cultured for $28 \mathrm{~d}$. Expression of (a) ColII, (b) ColX and (c) ColI in pellet, pellet-fibrin and upellet-fibrin constructs after 7 or $28 \mathrm{~d}$ of in vitro chondrogenic culture. Hypertrophy-associated gene COLX was upregulated in pellet and $\mu$ pellet-fibrin conditions cultured for $28 \mathrm{~d}$. 
stained in the fibrin encapsulated material (Fig. 6a). This background staining was far less intense than the one observed within our constructs. Staining specificity was shown in rat knee tissue. However, the optimal staining concentration for rat tissue was found to be lower than the one observed in human (Fig. 6d).

Generally, upellet-fibrin conditions had lower VEGF gene expression compared to pellet controls after $7 \mathrm{~d}$ of in vitro culture, an effect that was not observed after $28 \mathrm{~d}$ of culture (Fig. 7). In donor 1, after $28 \mathrm{~d}$ of culture, VEGF expression was slightly downregulated when compared to donors 2 and
3 (Fig. 7a). Other secreted factors, including BMP2 and MMP13, were differentially affected among conditions and donors, but were present in all samples. Downscaling the standard pellet to $\mu$ pelletfibrin size did not appear to hinder the expression of these markers (Fig. 7).

\section{$7 \mathrm{~d}$ of in vitro chondrogenic priming resulted in} tissue calcification in vivo

In addition to the in vitro characterisation, pellets, pellet-fibrin, $\mu$ pellets and $\mu$ pellet-fibrin constructs were subcutaneously implanted in nude mice for 8 weeks and harvested for volumetric and histological a.

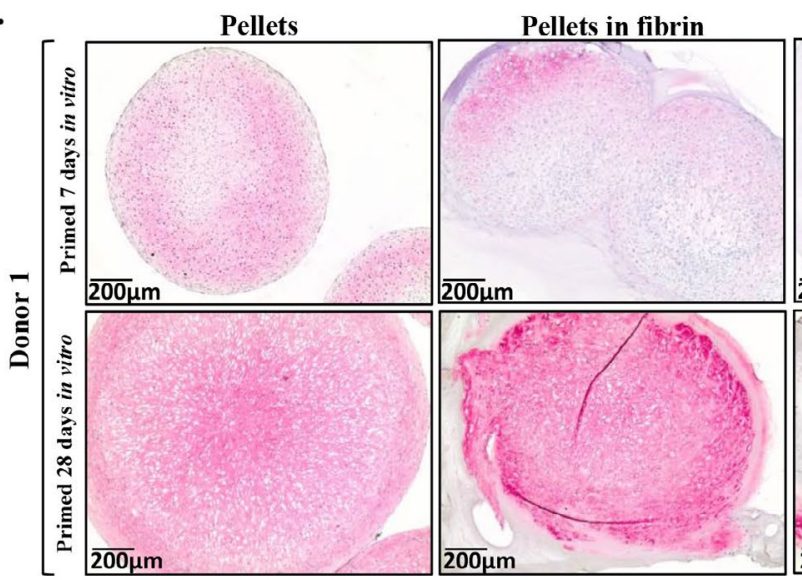

b.

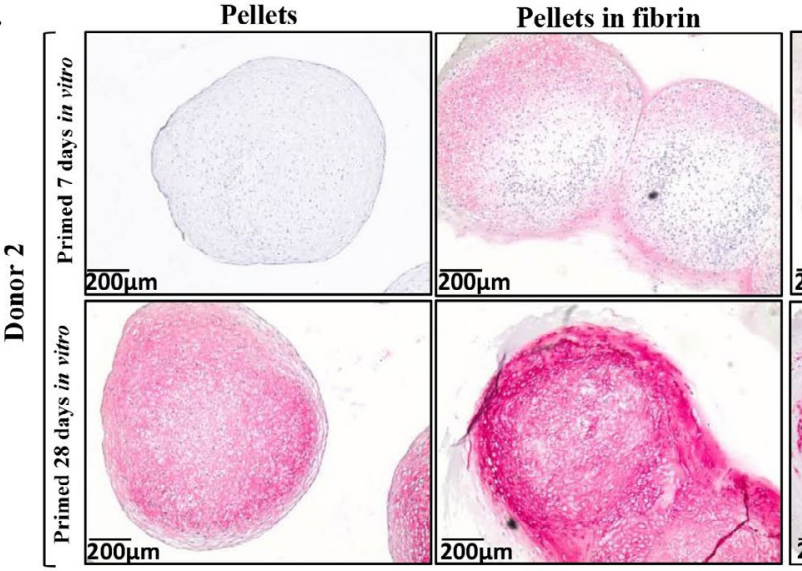

c.

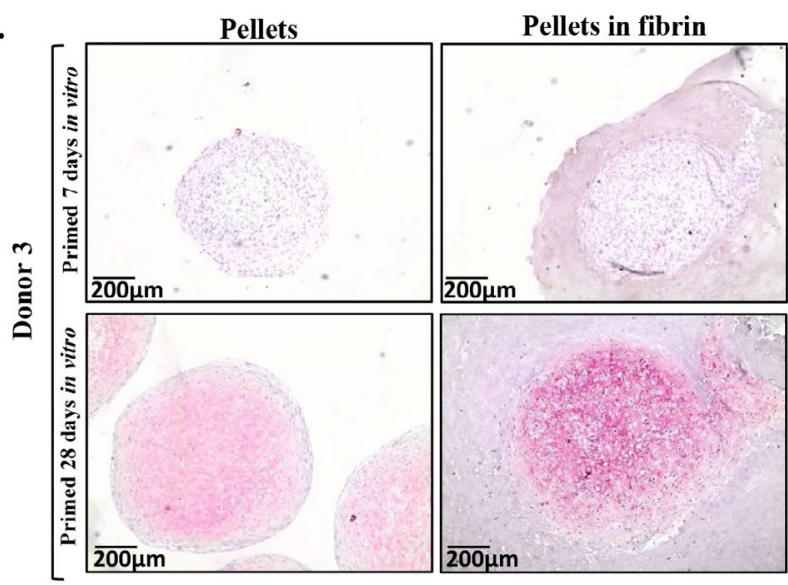

$\mu$ pellets in fibrin

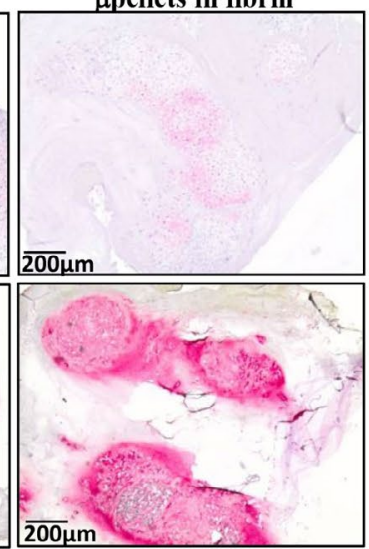

$\mu$ pellets in fibrin

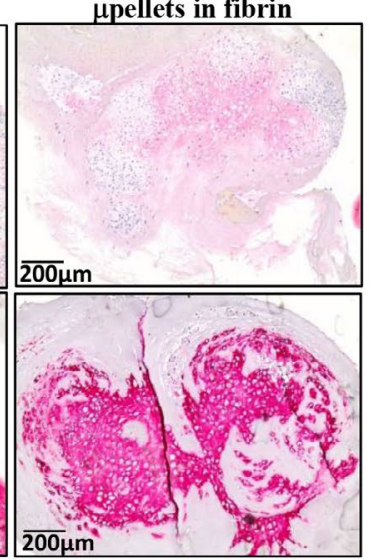

ppellets in fibrin

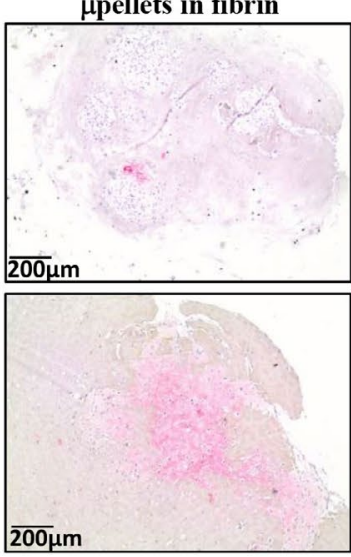

d.

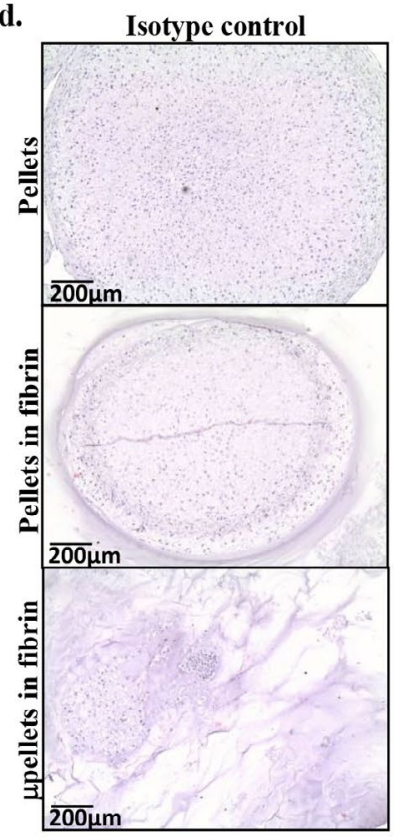

Fig. 5. upellet-fibrin constructs exhibited more homogenous collagen type II distribution after longer priming. COLII immunohistochemistry on chondrogenically differentiated pellet, pellet-fibrin and $\mu$ pelletfibrin constructs after 7 and $28 \mathrm{~d}$ of in vitro culture from MSCs of (a) donor 1, (b) donor 2 and (c) donor 3.(d) IgG control was performed on donor 1 samples collected at day 28. 
analysis (Fig. 8a). Non-encapsulated upellets could not be located following implantation and thus could not be analysed. Between the two in vitro priming times, 7 or $28 \mathrm{~d}$, there was significantly more calcified tissue present in conditions primed for $28 \mathrm{~d}$ in vitro, as shown by $\mu \mathrm{CT}$ analysis (Fig. 8b,c). Fibrin encapsulation of pellets lead to greater calcification in vivo than standard pellets, for all donors' MSCs cultured for $7 \mathrm{~d}$ (Fig. 8c). However, fibrin encapsulation did not appear to affect calcification when constructs were cultured for $28 \mathrm{~d}$. Pellets that were not encapsulated in fibrin were only retrieved from 1 donor after implantation following $7 \mathrm{~d}$ of in vitro culture and, for this donor, only 1 pellet of the 3 implanted was retrieved. Regardless of the donor, after in vivo implantation, $7 \mathrm{~d}$ primed pellet-fibrin and upellet-fibrin constructs were always retrieved and always showed calcification on $\mu \mathrm{CT}$ scans (Fig. 8b,c). $28 \mathrm{~d}$ primed pellets, pellet-fibrin and upellet-fibrin constructs were consistently retrieved from donors 1 , 2 and 3 and always showed comparable calcification among donors (Fig. 8b,c). In some donors, as shown in the $\mu \mathrm{CT}$ images (Fig. 8), pellets or $\mu$ pellets merged forming one larger construct. This was only observed a.

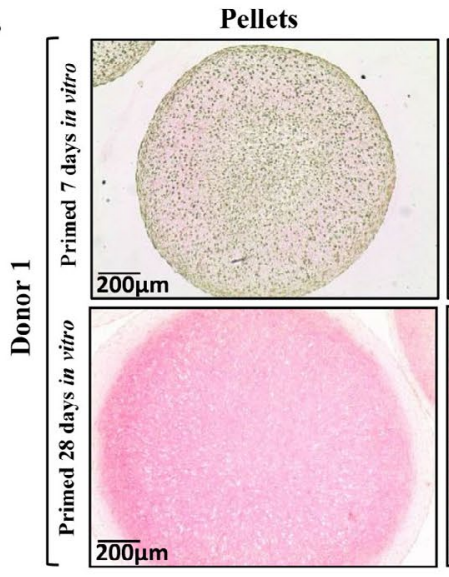

b.

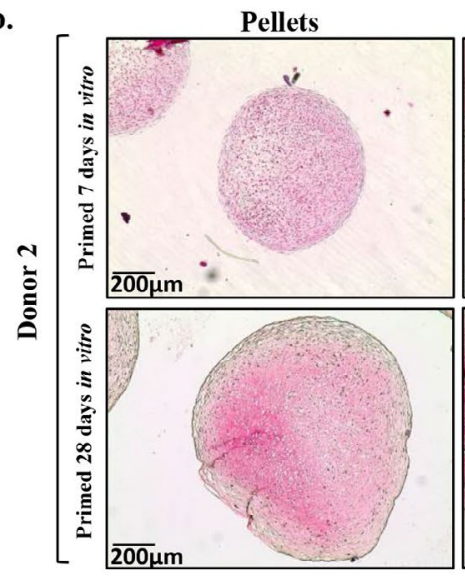

c.

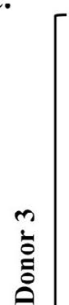

Pellets in fibrin

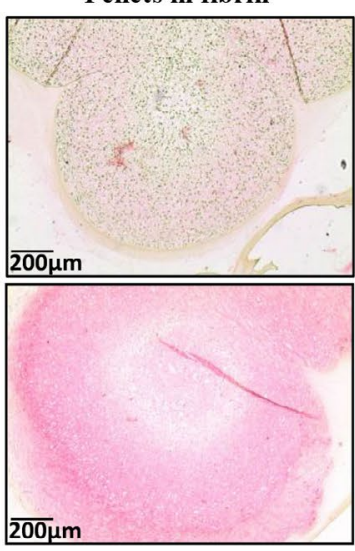

Pellets in fibrin

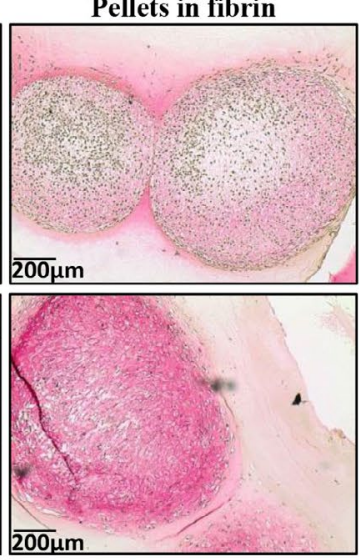

$\overline{200 \mu m}$

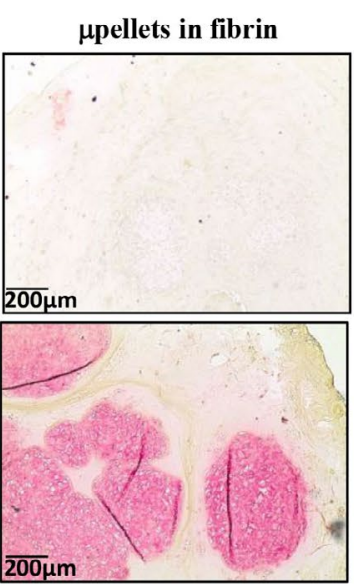

$\mu$ pellets in fibrin
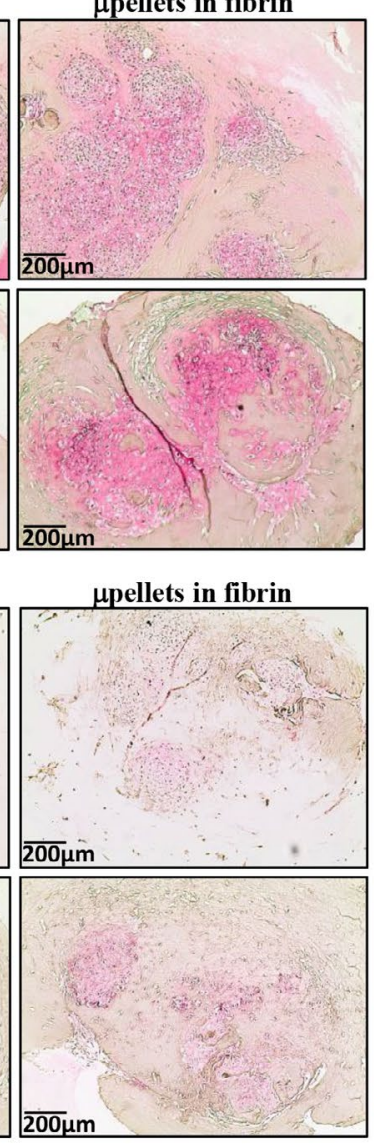

d.

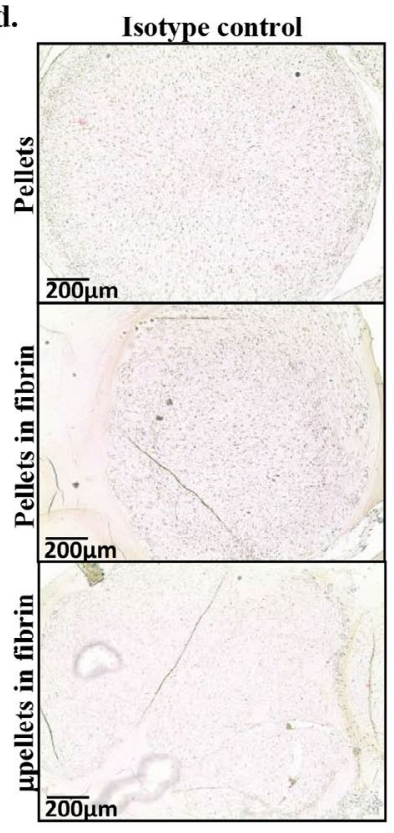

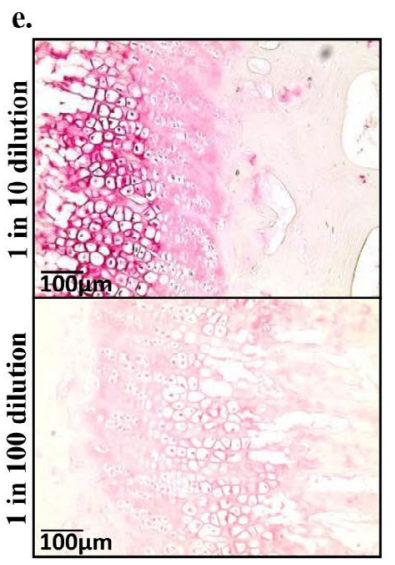

Fig. 6. Mpellet-fibrin constructs exhibited more homogenous collagen type $X$ distribution after longer priming. COLX immunohistochemistry on chondrogenically differentiated pellet, pellet-fibrin and upellet-fibrin constructs after 7 and $28 \mathrm{~d}$ of in vitro culture from (a) donor 1, (b) donor 2 and (c) donor 3. (d) IgG control was performed on donor 1 samples collected at day 28. (e) Rat knee positive control at $1: 10$ and $1: 100$ dilutions. 
within some of the fibrin-encapsulated samples and it was not observed consistently with a specific donor, priming time or condition.

Bone formation in vivo was dependent on extracellular matrix quality pre-implantation Bone and calcified cartilage were observed in all harvested samples. Calcified tissue was quantified by $\mu \mathrm{CT}$ (Fig. 9,10). However, non-encapsulated pellets primed in vitro for $7 \mathrm{~d}$ could only be retrieved

a.
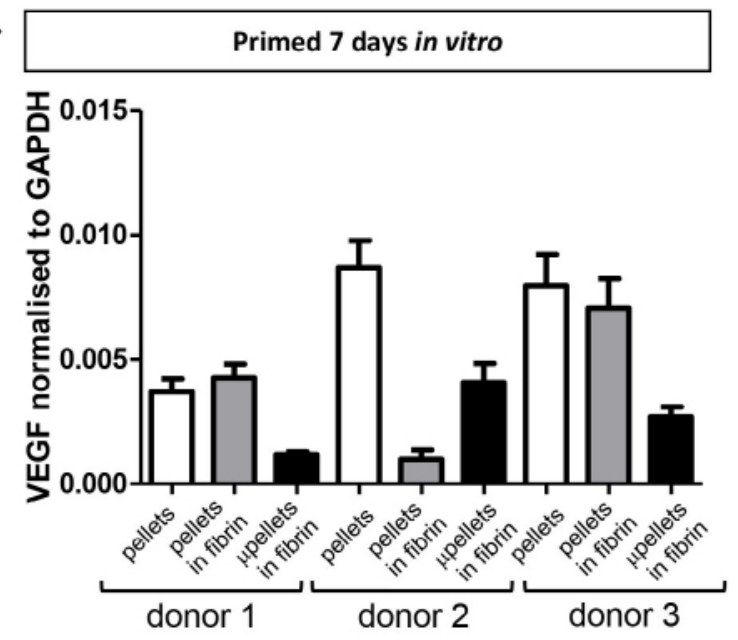

b.

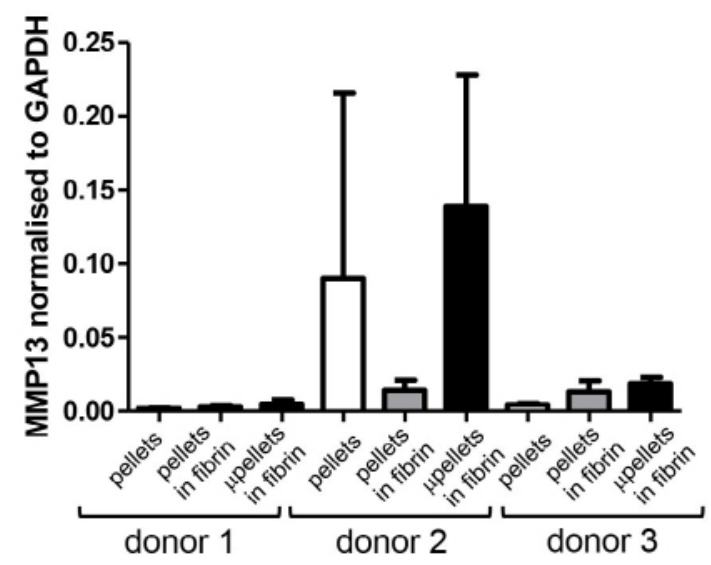

c.

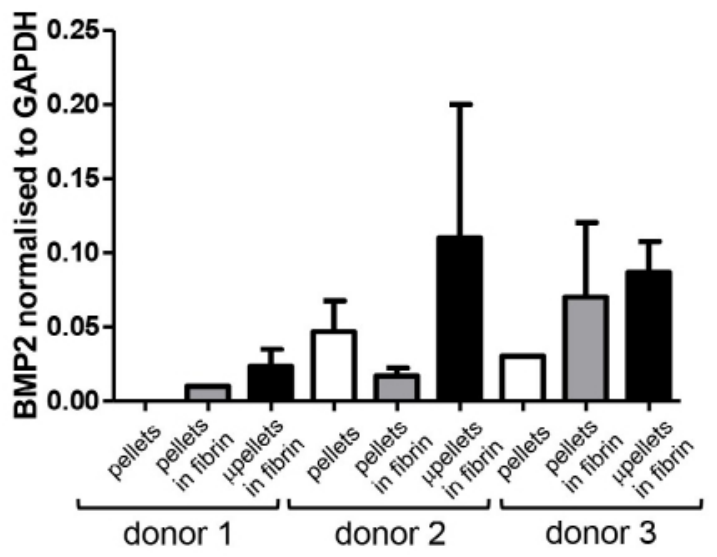

from donor 1 and the quantity of bone and calcified cartilage observed at the time of harvest in $H \& E$ sections varied among donors. Donor 1 had a denser GAG-rich matrix pre-implantation and higher COLII and COLX expression/production (Fig. 2,4,5,6). After 8 weeks in vivo, it resulted in an almost complete absence of bone marrow and only a small recruitment of matrix-remodelling osteoclasts (Fig. 9-11), compared to donor 2 and 3 that had a less GAG-rich matrix pre-implantation and lower COLII
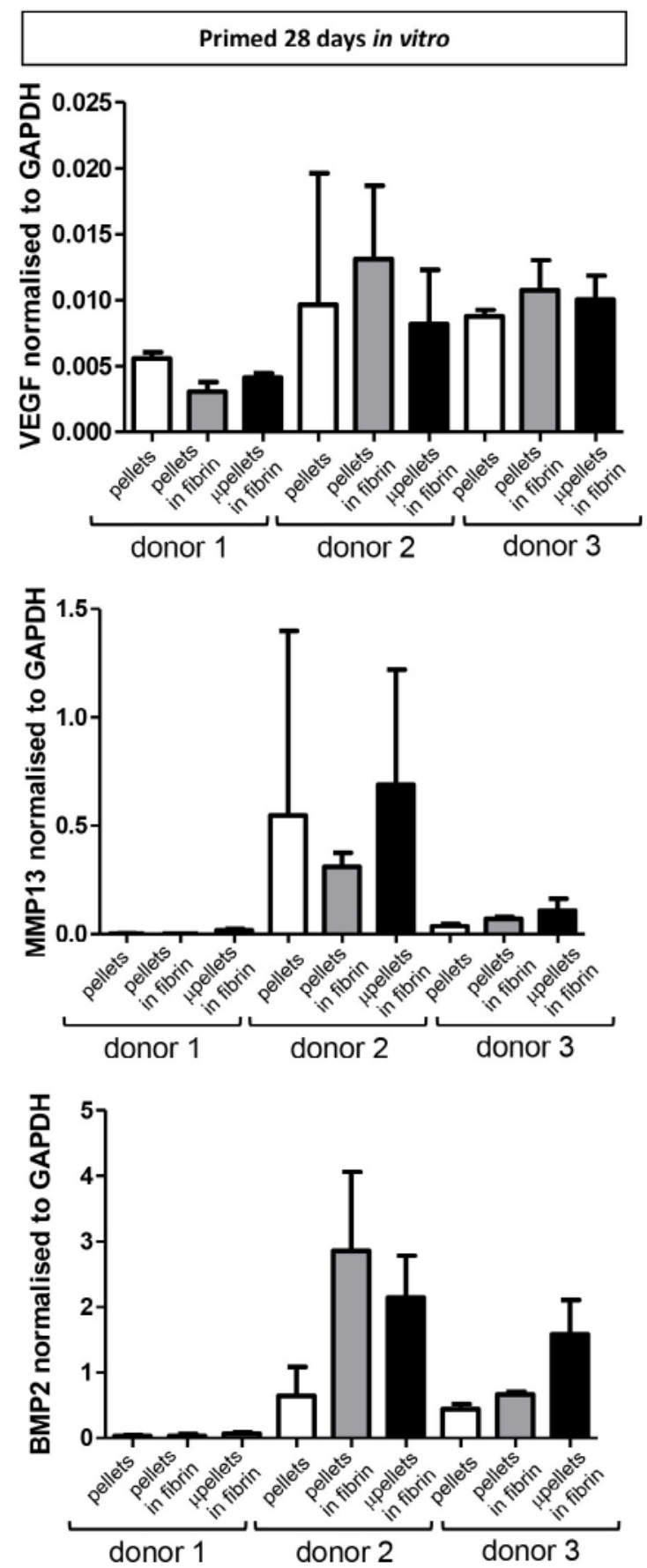

Fig. 7. Gene expression of secreted factors was differentially affected across donors and maintained in pellet-fibrin constructs. Expression of (a) VEGF, (b) MMP13 and (c) BMP-2 in pellet, pellet-fibrin and upellet-fibrin constructs after 7 or $28 \mathrm{~d}$ of in vitro chondrogenic culture. Hypertrophy-associated gene BMP2 was upregulated in pellet and $\mu$ pellet-fibrin conditions in some donors, whereas it was relatively unaffected in others. Other markers were differentially affected depending on the donor. 
a.

Day 0

Pellet formation

Day 1

encapsulation, chondrogenic culture

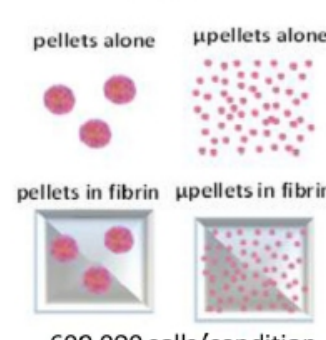

600,000 cells/condition

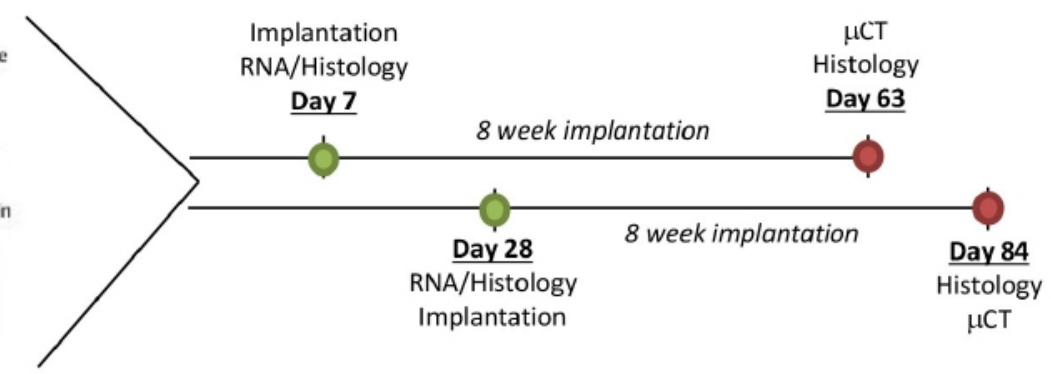

b.
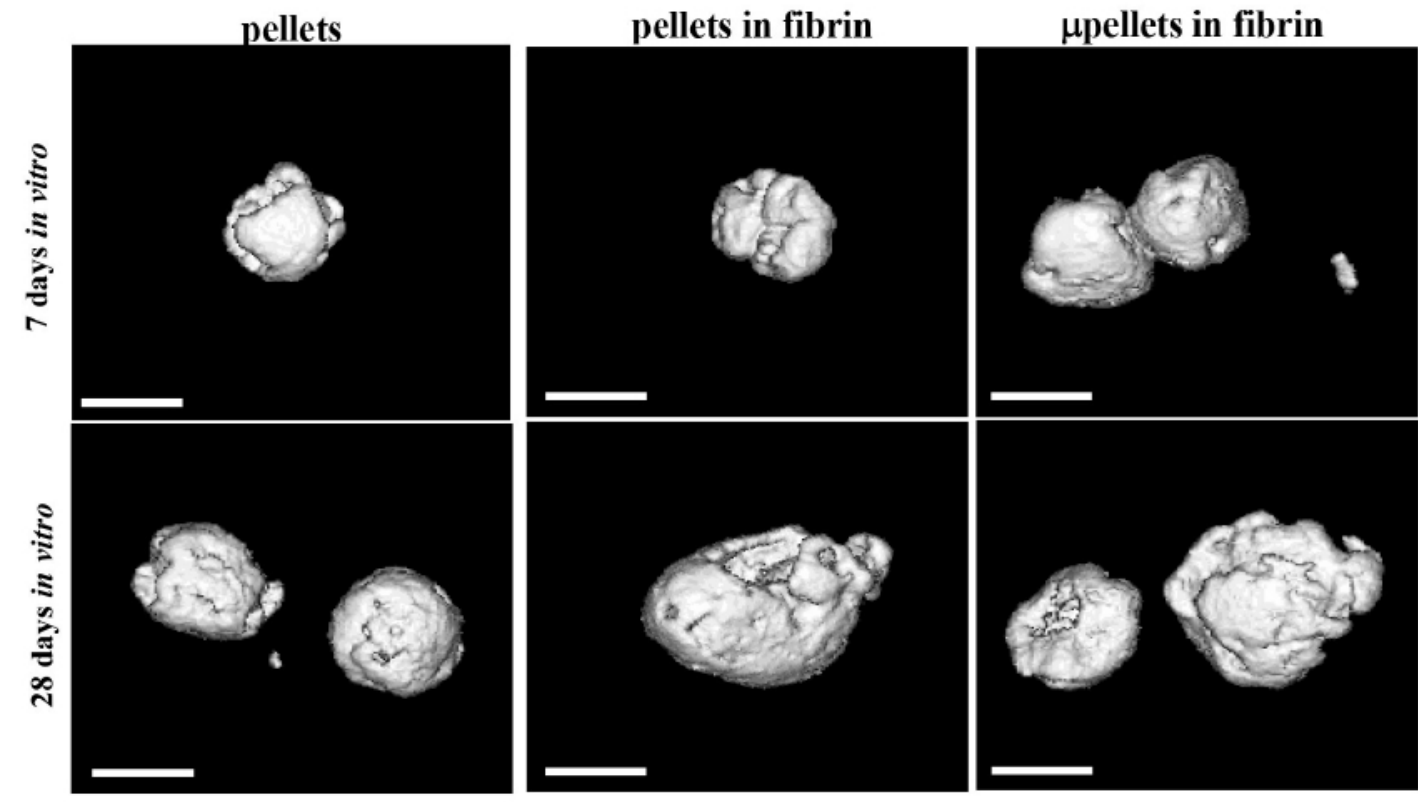

c.
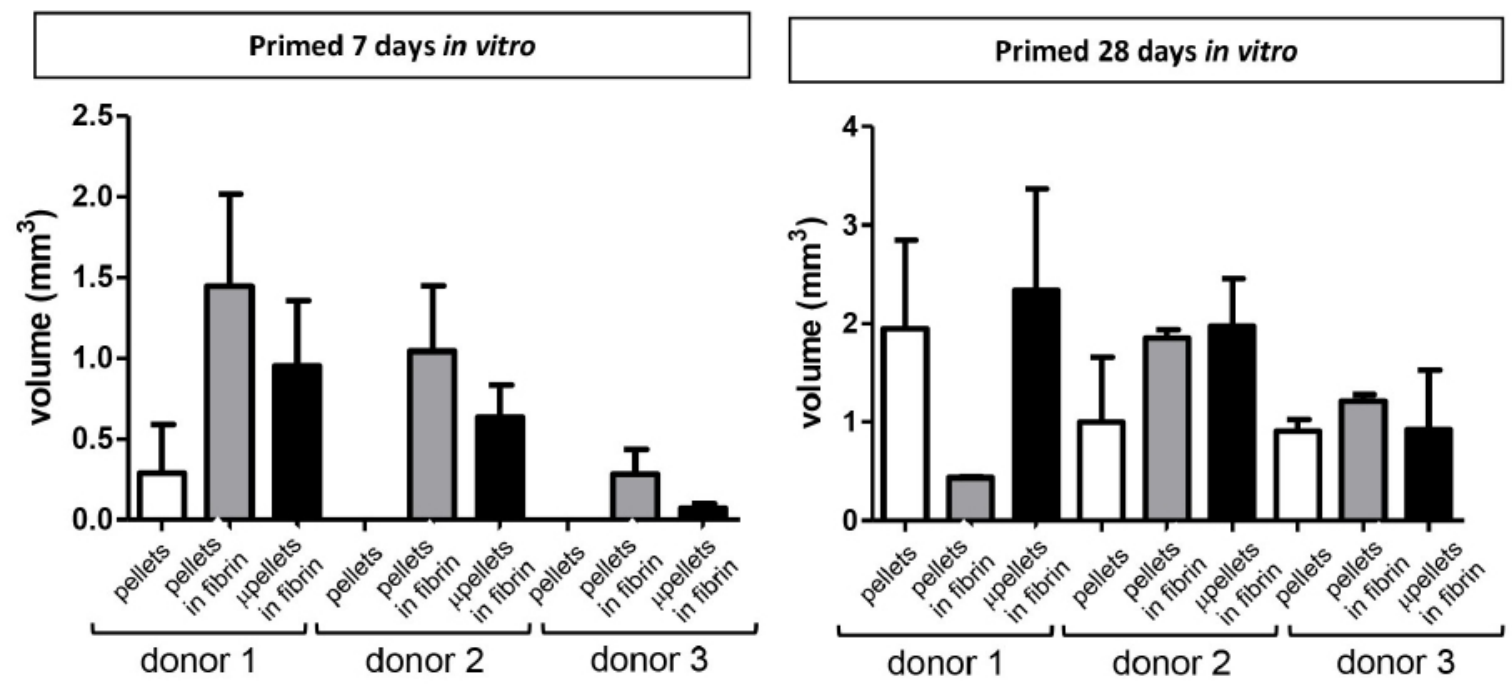

Fig. 8. $7 \mathrm{~d}$ primed samples and $\mu$ pellet constructs formed mineralised tissue after in vivo implantation. (a) In vivo study outline, (b) $\mu \mathrm{CT}$ scans of constructs primed in vitro for 7 or $28 \mathrm{~d}$ after 8 weeks of in vivo implantation. (MSC from donor 1; the white bar represented an arbitrary length, for comparison among $\mu \mathrm{CT}$ images). (c) Volume of mineralised tissue obtained from ex vivo $\mu \mathrm{CT}$ scans showed donor-related differences in mineralisation. 
and COLX expression/production (Fig. 2,4,5,6). This resulted in a higher percentage of bone marrow elements and greater osteoclast recruitment, after implantation in vivo for a comparable time (Fig. $9,10,12,13)$. These differences were observed most clearly in the histological sections, because it was not possible, in the $\mu \mathrm{CT}$, to distinguish between calcified cartilage from bone.

\section{Discussion}

Priming time and reproducible bone formation represent two of the major hurdles for translation of TE based approaches to the clinic. In this manuscript, we demonstrated that $7 \mathrm{~d}$ of chondrogenic priming coupled with encapsulation in a fibrin-gel was sufficient to allow bone formation in vivo. Following
$7 \mathrm{~d}$ of in vitro priming, pellets consistently produced a more chondrogenic GAG-rich matrix and hypertrophic markers were consistently upregulated. However, without encapsulation, only MSCs from one donor were observed to calcify in vivo after being primed for $7 \mathrm{~d}$ in vitro. The same MSCs produced no bone marrow elements. Given the lack of bone formation, the chondrogenic markers we chose were most likely insufficient predictors of in vivo bone formation.

Novel $\mu$ pellet-fibrin constructs were created and characterised to ensure that downscaling the standard pellet culture to a $\mu$ pellet format would not inhibit chondrogenesis and subsequent bone formation. We observed that $\mu$ pellet-fibrin constructs showed equivalent matrix production and bone formation compared to standard pellet culture counterparts. In a recent predictive model, it is shown
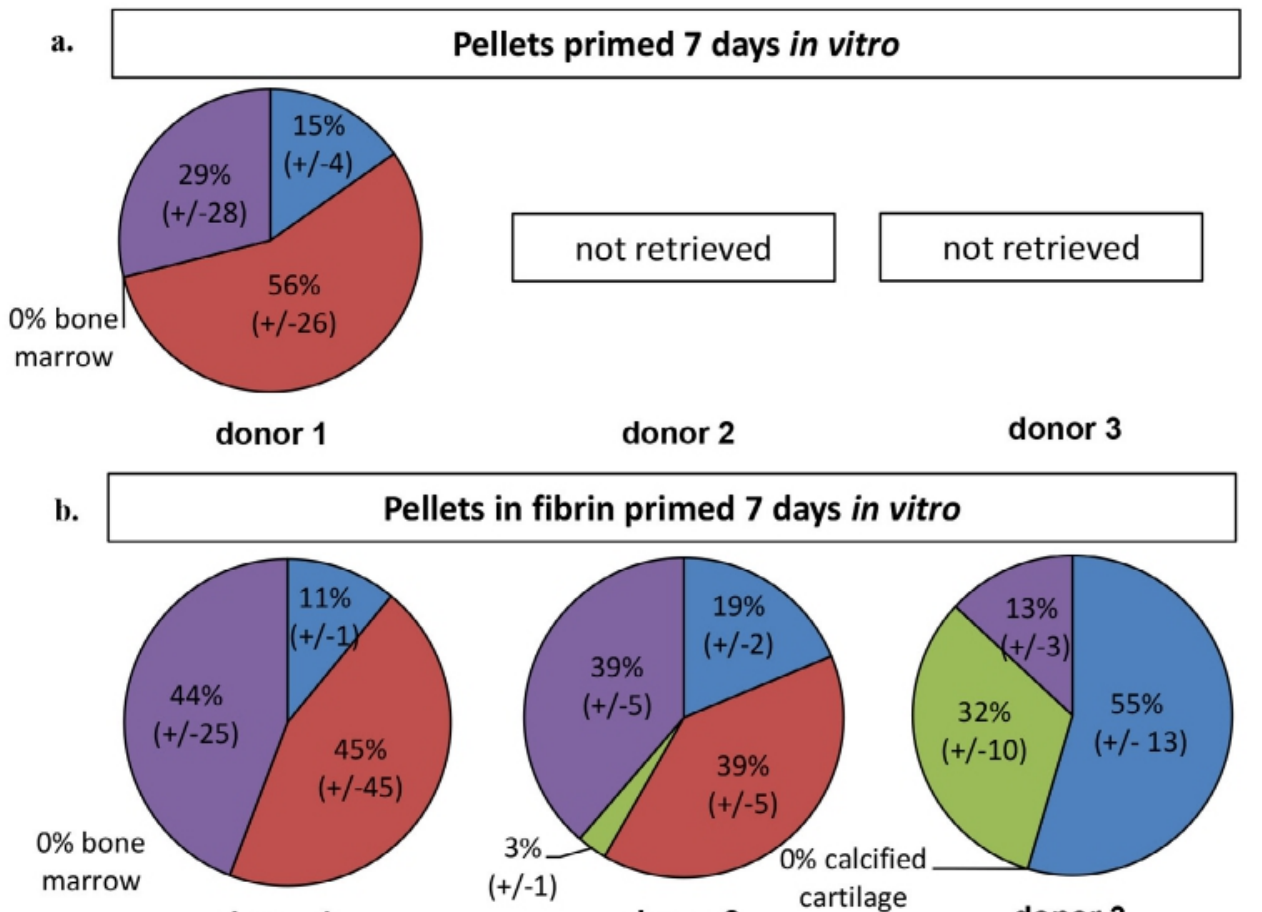

donor 1

$(+/-1)$

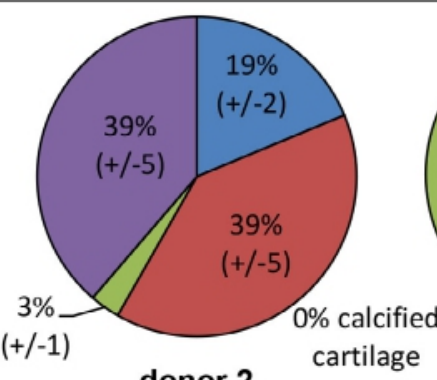

donor 2

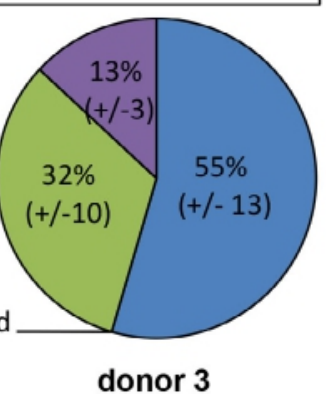

donor 3

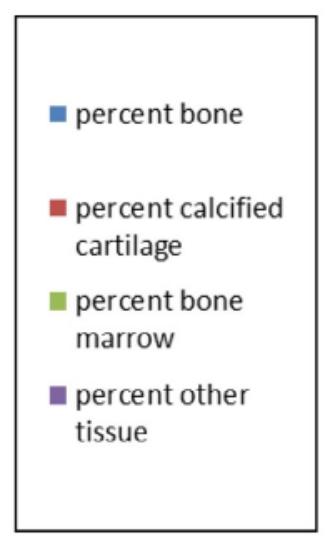

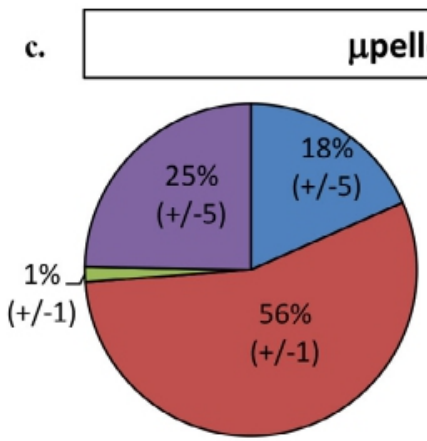

donor 1

upellets in fibrin primed 7 days in vitro

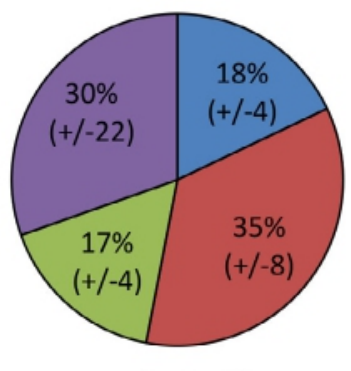

donor 2

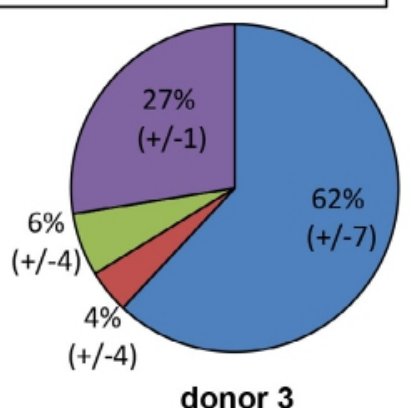

donor 3

Fig. 9. Quantity of bone and bone marrow following $7 \mathrm{~d}$ of in vitro priming was donor-dependent. Percentage of bone, bone marrow, calcified cartilage and other tissue found in samples primed in vitro for $7 \mathrm{~d}$ following 8 weeks in vivo. Quantification of (a) pellets, (b) pellet-fibrin and (c) $\mu$ pellet-fibrin (3 constructs per condition and donor; 3 MSC donors used) was completed using ImageJ and high magnification (200×) images of H\&E staining and segmenting areas, based on morphology. 
that matrix deposition and collagen distribution tend to accumulate around the outer periphery of pellets, where nutrient supply is abundant (Lewis et al., 2016). They predicted that uniform distribution of proteoglycans and collagens could be maintained by decreasing pellet size, which would make nutrients and factors more available to individual differentiating cells. Indeed, this could explain why we saw an increase in some matrix-related genes in $\mu$ pellet constructs. In addition, decreased VEGF gene expression in $\mu$ pellet-fibrin constructs, compared to standard pellets, was observed after $7 \mathrm{~d}$ of culture, but this decrease was not observed after $28 \mathrm{~d}$ of in vitro priming and did not appear to hinder in vivo bone forming capacity in $\mu$ pellet-fibrin constructs.

In vivo results confirmed that bone formation could be achieved by chondrogenically priming constructs for only $7 \mathrm{~d}$ in vitro using either standard or upellet-fibrin constructs. Non-encapsulated $\mu$ pellet constructs failed to differentiate in all donors and time points, except for non-encapsulated $\mu$ pellets from donor 3 cultured for $28 \mathrm{~d}$. These non-encapsulated upellets aggregated, forming one pellet after $28 \mathrm{~d}$ of culture. This aggregated pellet and all other nonencapsulated $\mu$ pellets that were implanted did not result in bone formation, indicating that $\mu$ pellets alone and aggregated $\mu$ pellets were not capable of bone formation. It appeared that $\mu$ pellets required encapsulation, during in vitro differentiation or during implantation, in order for bone formation to be achieved. We observed that fibrin encapsulation increased bone formation when priming time was reduced to $7 \mathrm{~d}$ in subcutaneously implanted pellet constructs. $7 \mathrm{~d}$ non-fibrin-encapsulated pellets were rarely retrieved (one in three donors) and all retrieved pellets lacked bone marrow elements. Given the lack of bone formation, it is probable that other chondrogenic markers may more accurately predict
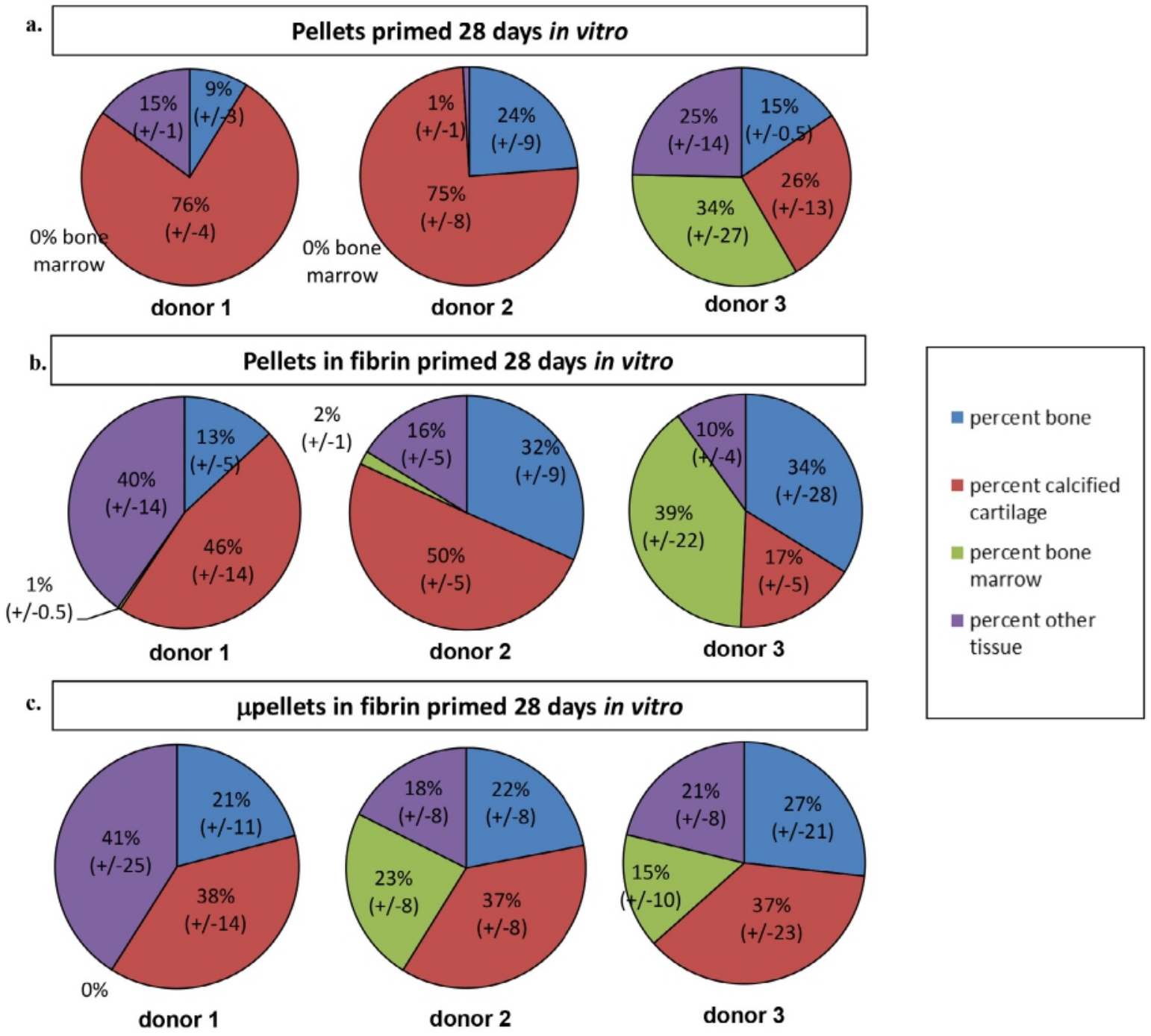

Fig. 10. Quantity of bone and bone marrow following $28 \mathrm{~d}$ of in vitro priming was donor-dependent. Percentage of bone, bone marrow, calcified cartilage and other tissue found in samples primed in vitro for 28 d following 8 weeks in vivo. Quantification of (a) pellets, (b) pellet-fibrin and (c) upellet-fibrin (3 constructs per condition and donor; 3 MSC donors used) was completed using ImageJ and high magnification (200×) images of H\&E staining and segmenting areas, based on morphology. 
in vivo bone formation than the ones we selected. It is possible that pellets that did not calcify or form bone were simply absorbed by the host or, perhaps, the addition of fibrin prevented this absorption long enough for remodelling and bone formation to take place.

Often, fibrin-encapsulated samples exhibited fusion in vivo, resulting - in the $\mu \mathrm{CT}$ scans - in a fused mass of pellets/ $\mu$ pellets when compared to individual non-encapsulated pellets. This fusion was not consistently observed among conditions or donors, but was only observed in fibrin-encapsulated conditions. It could be that the fibrin contracted bringing the pellets in closer proximity and allowing for this fusion to take place. Fibrin has been shown to reduce oxygen diffusion and tension, leading to a hypoxic environment (Demol et al., 2011). This hypoxic environment is favourable for bone formation and has been shown to increase osteoclast and osteoblast activity. This may explain how fibrin is able to enhance bone formation in vivo (Arnett et al., 2003; Hung et al., 2012; Steinbrech et al., 1999). However, in a prior pilot study, pellets primed in vitro for $7 \mathrm{~d}$ in the absence of fibrin did form bone containing mature marrow cavities (data not shown). This highlights a degree of variability in graft performance based on inherent donor differences, which have been previously reported (Amini et al., a.

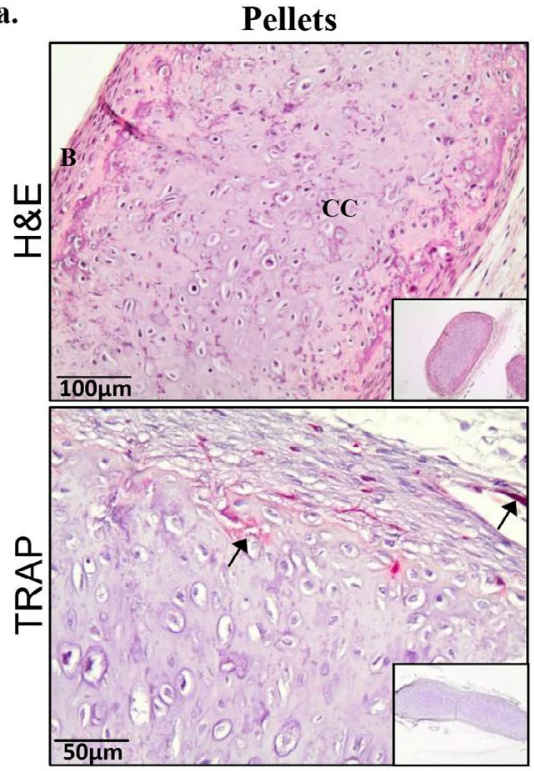

b.

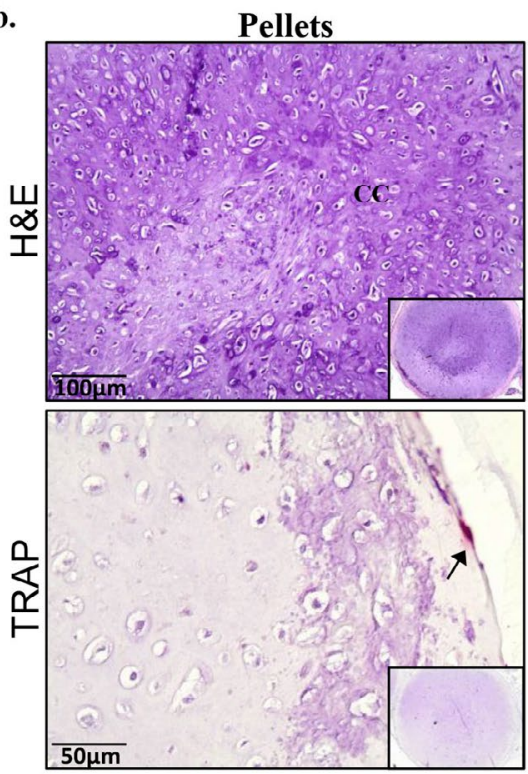

Pellets in fibrin

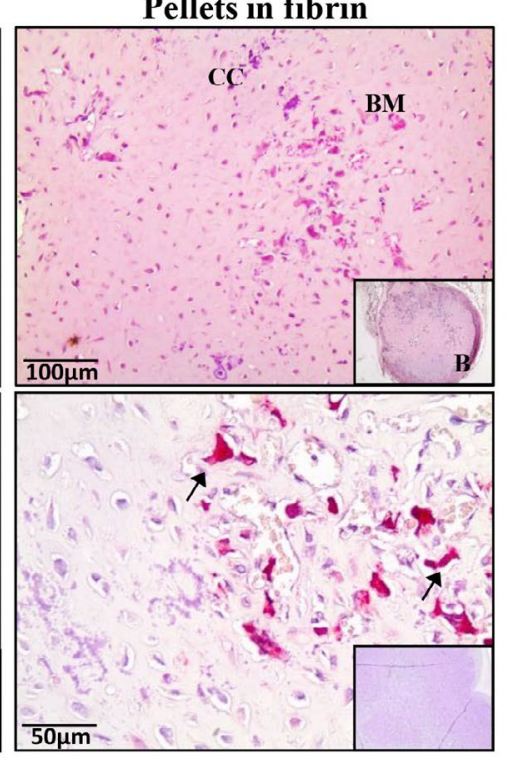

Primed 28 days in vitro Pellets in fibrin

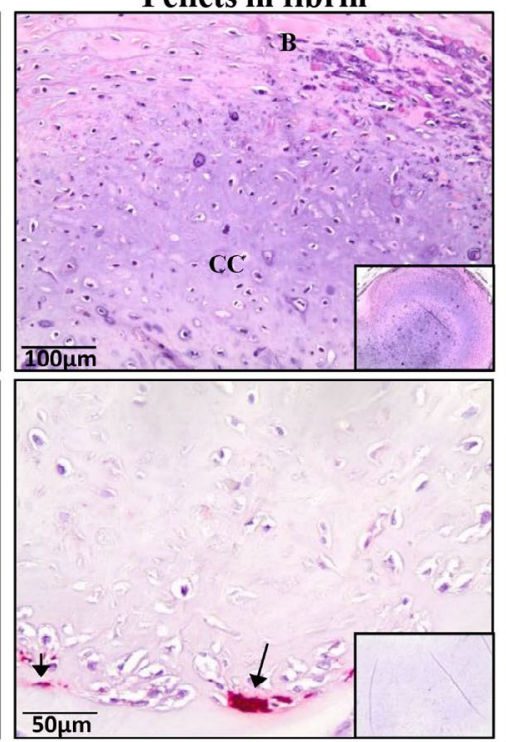

$\mu$ pellets in fibrin

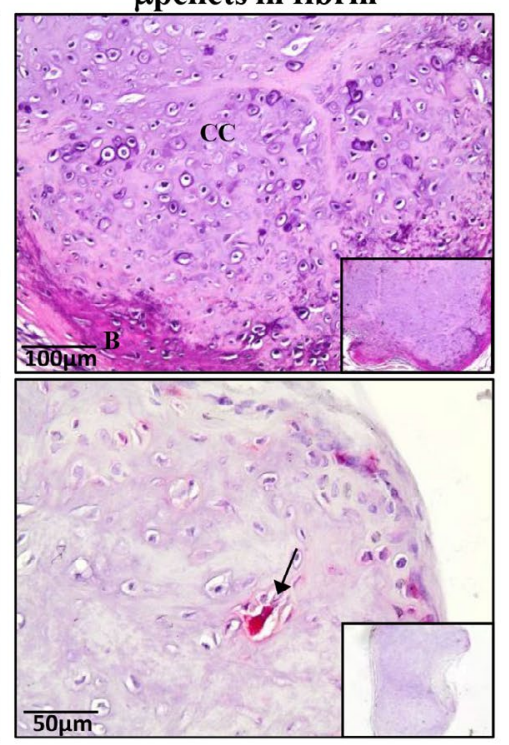

$\mu$ pellets in fibrin

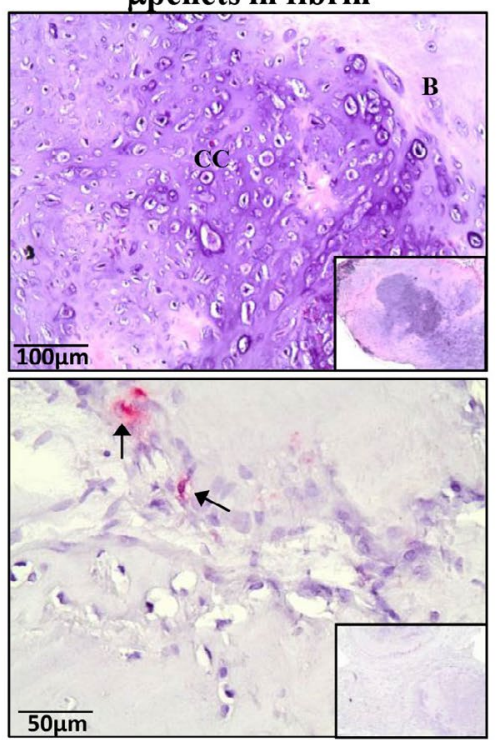

Fig.11. High GAG density in vitro resulted in slower remodelling and bone formation in vivo. (a) Day 7 and (b) day 28 in vivo H\&E and TRAP stainings of donor 1 . The intensity of the safranin O staining (Fig. 2) showed that the quantity of GAGs was high pre-implantation. Denser GAG-rich matrices appeared to be harder for cells to penetrate, as evident by a decrease in bone marrow formation and osteoclast infiltration (in H\&E stains CC = calcified cartilage, BM = bone marrow, B = bone; in TRAP staining arrows indicate areas of TRAP positivity). 
2012; van der Stok et al., 2014). Indeed, in this study we observed that major differences among donors influenced the degree of bone formation observed following implantation.

In our study, we observed a difference in chondrogenic differentiation and subsequent bone formation among all three donors. Samples retrieved from donor 1 often lacked bone marrow elements following implantation. In vitro analysis from donor 1 showed a decrease in both VEGF and COLX gene expression compared to the other two donors. VEGF is known to be involved in vascularisation
(Yang et al., 2012), which means that this decreased expression of VEGF could be attributed to the lack of bone marrow elements we observed following in vivo implantation. Additionally, donor 1 showed greater COLII expression and GAG accumulation, as well as, decreased COLX gene expression compared to donors 2 and 3. Histologically, we observed that GAG and other matrix collagens production pre-implantation appeared to be related to bone formation in vivo. A denser matrix, containing more collagen elements and GAGs, resulted in less mature bone formation, more incomplete remodelled a.

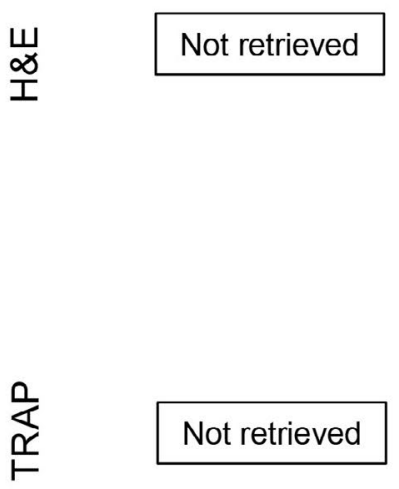

b.

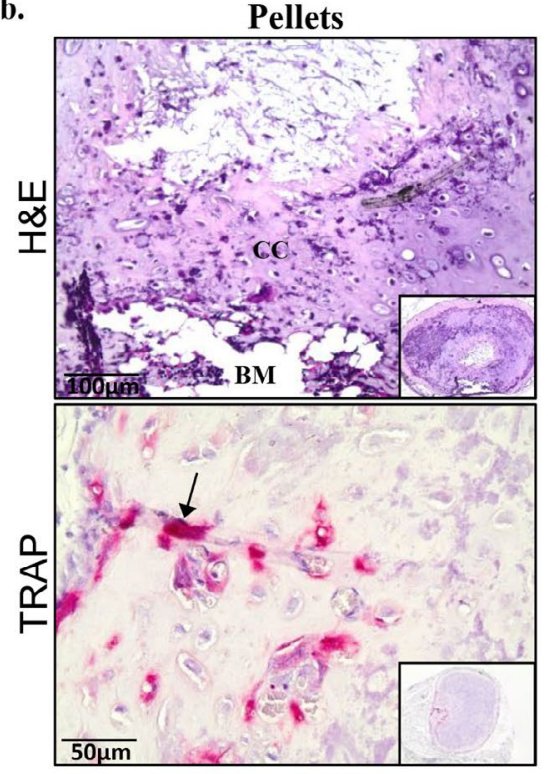

Primed 7 days in vitro

Pellets in fibrin
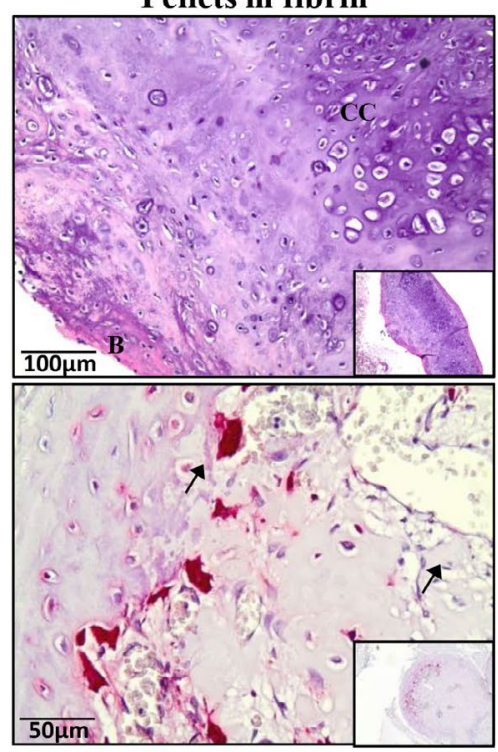

Primed 28 days in vitro

Pellets in fibrin

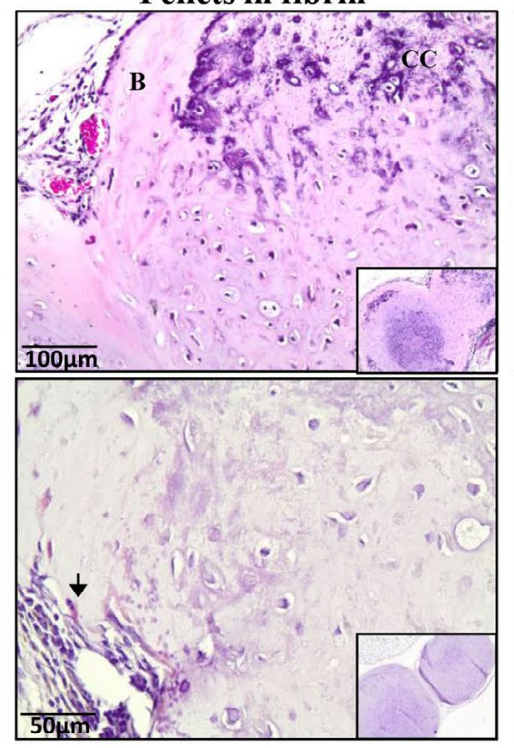

Hpellets in fibrin
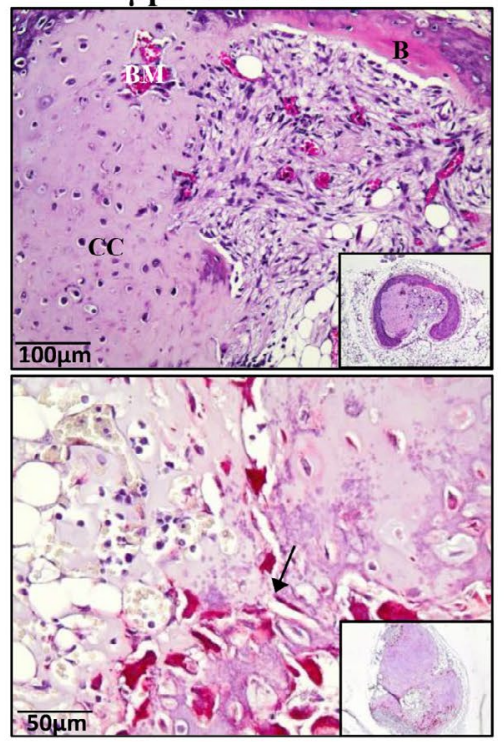

$\mu$ pellets in fibrin

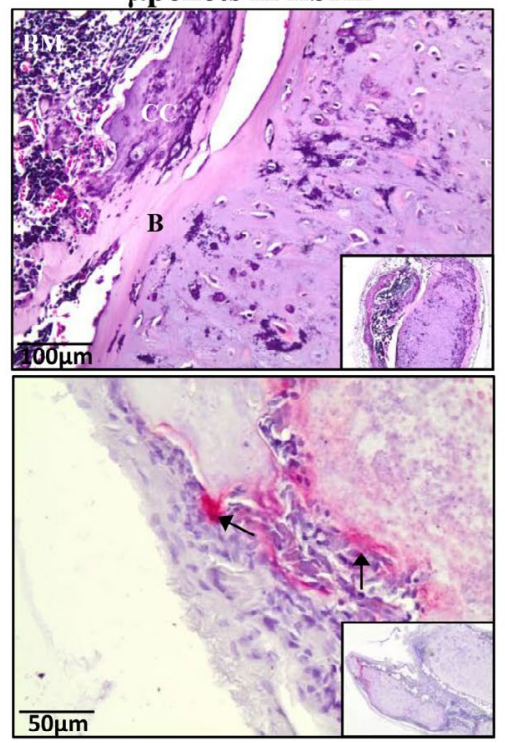

Fig. 12. GAG density in vitro affected the rate of remodelling and bone formation in vivo. (a) Day 7 and (b) day 28 in vivo H\&E and TRAP stainings of donor 2. The intensity of the safranin O staining (Fig. 2) showed a moderate quantity of GAGs pre-implantation. In vivo there was a varying degree of calcification, bone marrow space development and osteoclast infiltration, which appeared to be correlated to in vitro GAG deposition pre-implantation (in H\&E stains CC = calcified cartilage, $\mathrm{BM}=$ bone marrow, $\mathrm{B}=\mathrm{bone}$; in TRAP staining arrows indicate areas of TRAP positivity). 
calcified cartilage and a decrease in the presence of matrix remodelling osteoclasts. These differences were best observed in the H\&E stained sections, as the $\mu \mathrm{CT}$ - quantifying the entire area of calcified tissues - were unable to distinguish between quantities of bone and calcified cartilage. We hypothesised that denser matrices took longer to be remodelled in vivo, as vessel and cell invasion were more difficult in a dense environment, which resulted in delayed bone formation. On the contrary, a less dense matrix could be invaded more easily, leading to faster vascularisation and remodelling, thus resulting in quicker bone formation. Supporting our hypothesis, it has previously been shown that GAG depletion in articular cartilage leads to an increase in blood vessel invasion, which contributes to osteoarthritis and bone development in diseased cartilage (Akkiraju and Nohe, 2015; Connelly et al., 2008). However, we also hypothesised that a minimum level of matrix production was necessary for bone formation to occur and to prevent pellets from being resorbed or lost in vivo, as evidenced by the fact that $7 \mathrm{~d}$ primed pellets - that had extremely low levels of GAG production pre-implantation - were unable to a.

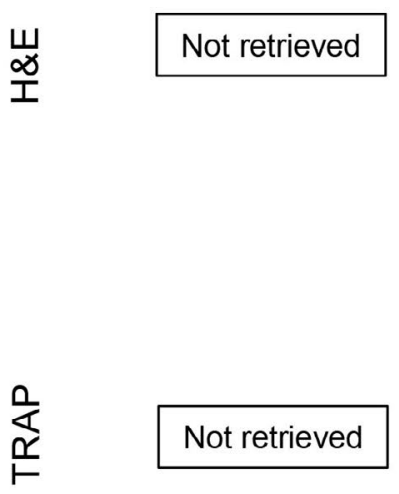

Pellets
Primed 7 days in vitro Pellets in fibrin

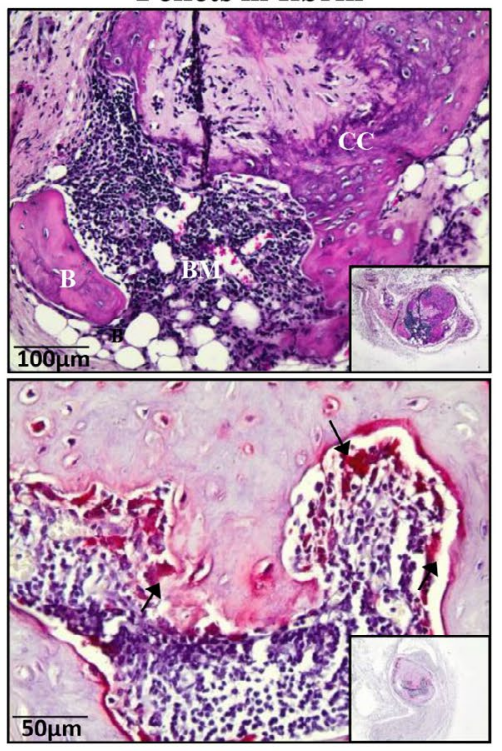

Primed 28 days in vitro

Pellets in fibrin

b.
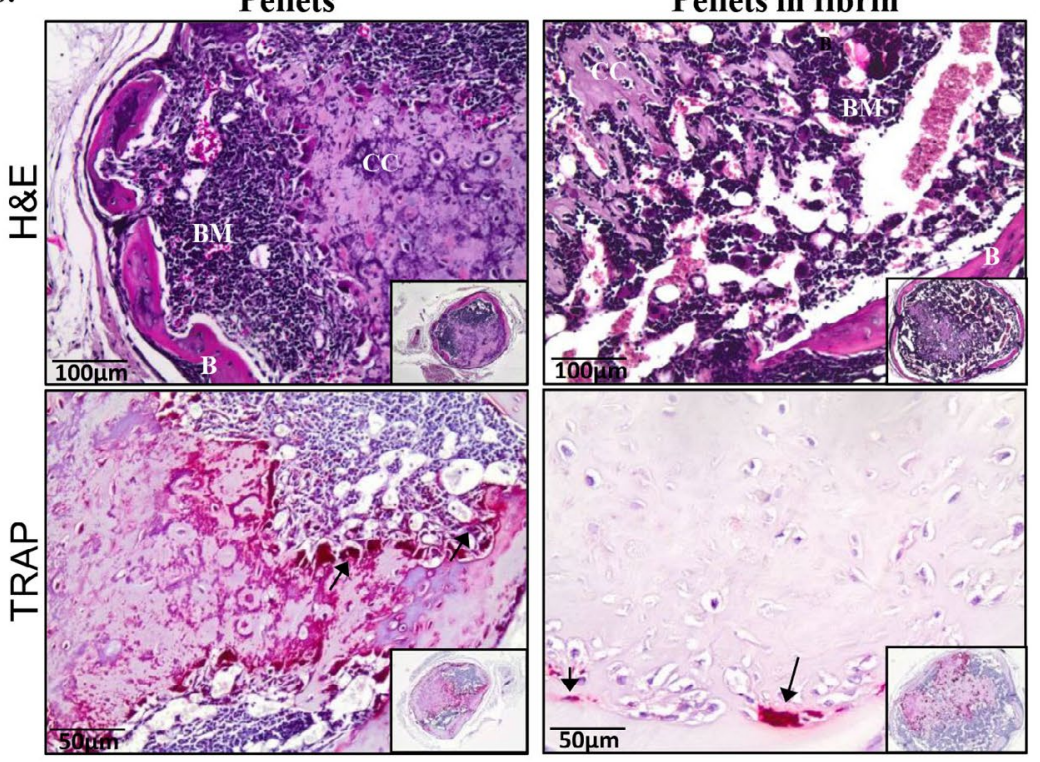

$\mu$ pellets in fibrin

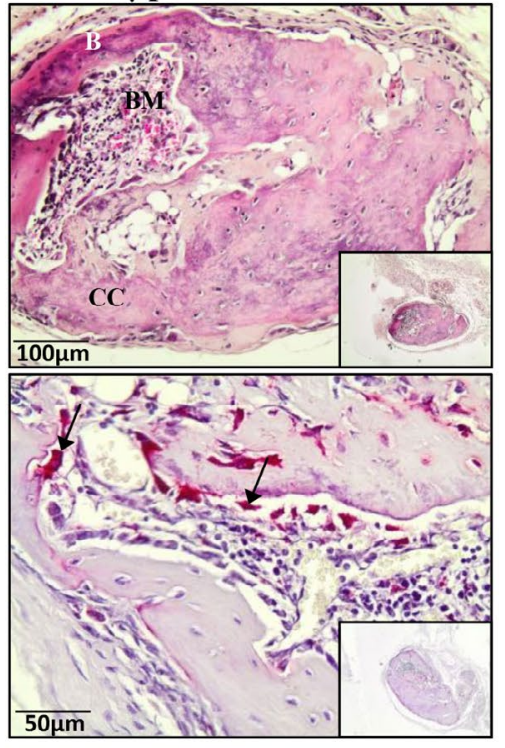

$\mu$ pellets in fibrin

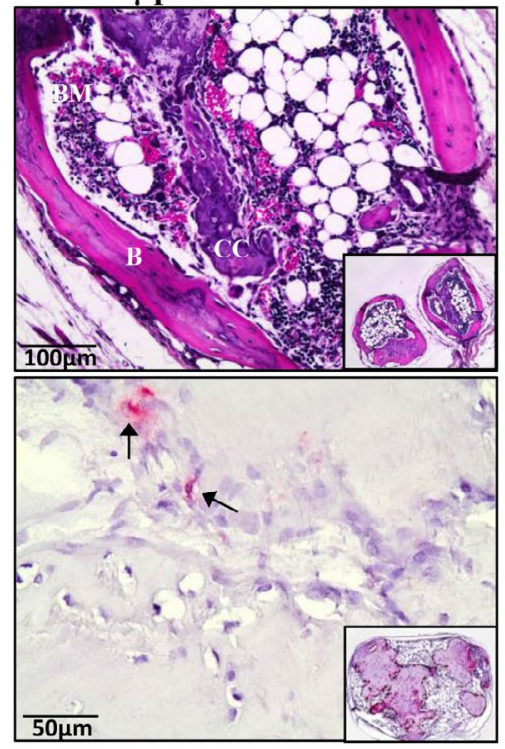

Fig. 13. Lower GAG density in vitro resulted in accelerated remodelling and bone formation in vivo. (a) Day 7 and (b) day 28 in vivo H\&E and TRAP staining for donor 3. The intensity of the safranin O staining (Fig. 2) showed that the quantity of GAGs was low pre-implantation. Less dense GAG-rich matrices appeared to be easier for cells to penetrate, as evident by an increased in bone marrow formation and osteoclast infiltration (in H\&E stains CC = calcified cartilage, BM = bone marrow, B = bone; in TRAP staining arrows indicate areas of TRAP positivity). 
be retrieved (donor 2 and 3). It is difficult to say if variation in matrix elements alone could justify the difference we observed in bone formation. In order to properly investigate this aspect, a larger number of donors - which we had not access to - needs to be investigated in future studies.

It is also possible that the model we used could have hindered or slowed matrix remodelling of more dense matrices, such as T-lymphocytes, known to degrade GAGs in vivo (Savion et al., 1984), were absent from our model. If these T-lymphocytes had been present, faster bone formation from samples with a more GAG-rich matrix may have been observed. However, this would require an immunocompetent model in order to be verified. We have used immunocompromised mice, lacking functioning $\mathrm{T}$ - and B-cells, a model which is often employed in ectopic bone formation studies (Jukes et al., 2008; Scotti et al., 2013). Our group has previously shown that it is possible to take chondrogenically primed MSCs from rats and implant them into immunocompetent animals to achieve bone formation (Farrell et al., 2011). Whether human MSCs would form bone in an immunocompetent animal has yet to be determined. As we implanted human MSC pellets in a mouse environment, we utilised an immunodeficient mouse model to avoid xenographic rejection. Because this study was a proof of principle study to characterise and determine the de novo bone forming potential of the upellet-fibrin constructs, we implanted our constructs in a subcutaneous environment - which we felt gave the best conditions to answer our question. We chose this instead of a large bone defect model, as we were better able to adhere to the 3R principle (Kilkenny et al., 2011), i.e. safely implanting more conditions per animal allowing for fewer animals with minimal discomfort to be used to complete the study. Subcutaneous implantation of our relatively small constructs did not hinder their movement or range of motion. Constructs were not in an environment where bone forms naturally and this might have limited the bone formation and remodelling we observed within our constructs. Future studies investigating these constructs in a defect environment will be useful.

upellet-fibrin constructs were found to have equivalent calcification volumes (as assessed by $\mu \mathrm{CT}$ ) after in vivo implantation, when compared to pellets cultured for the same time. These volumes, even after $28 \mathrm{~d}$ of culture pre-implantion, were still far from being sufficient to properly heal a large bone defect. As we have not studied how these constructs perform in a large defect, we cannot say how they would calcify and integrate with the host bone tissue. Furthermore, the inherent donor differences we observed meant that although samples were primed for the same time, the ultimate bone formation varied after implantation. This donor variation is an area of active research and furthering our understanding of what is crucial for endochondral bone formation to take place in vivo, in response to certain in vitro produced cues, is essential for this research to move forward. In addition, there is often a lack of bridging between standard pellets, which needs to be addressed for proper integration of such constructs with host tissue. Scaled-up approaches are still an area of active research in this field and in the future we hope to investigate these $\mu$ pellets using bioactive injectable matrices in large bone defects to increase bone formation. Additionally, understanding the relationship between matrix composition preimplantation and the subsequent effect on bone formation in vivo is important and merits further investigation, as determining an optimal set of criteria for chondrogenesis pre-implantation to achieve bone formation in vivo will improve future research. This is also important because identifying such criteria would result in more reproducible outcomes, which is crucial for the translation of such constructs to the clinic. Still we find the bone forming potential of these novel upellet constructs to be promising. We believe that, with further optimisation of in vitro culture, we could optimise these constructs as an injectable bone construct, which would be advantageous to treat bone defects.

\section{Conclusions}

This study showed that it was possible to decrease significantly in vitro culturing for pellet and $\mu$ pellet constructs and still retrieve bone after implantation. After further optimisation of culture conditions and material parameters, $\mu$ pellets could offer a promising alternative to current clinical treatment options for bone defects. Also, we believe that upellets, due to their small size, could be easily optimised as an injectable therapy using either a thermoreversible gel or injectable fibrin-based material, allowing for the creation of a customisable void filling bone substitute. Given the shorter priming time required, coupled with their injectability, this approach could be a promising minimally-invasive therapy for replacing some autologous bone transplantation procedures.

\section{Acknowledgements}

We would like to thank Nicole Kops from the Department of Orthopaedics at Erasmus MC for her technical support.

\section{References}

Akkiraju H, Nohe A (2015) Role of chondrocytes in cartilage formation, progression of osteoarthritis and cartilage regeneration. J Dev Biol 3: 177-192.

Amini AR, Laurencin CT, Nukavarapu SP (2012) Bone tissue engineering: recent advances and challenges. Crit Rev Biomed Eng 40: 363-408. 
Arnett TR, Gibbons DC, Utting JC, Orriss IR, Hoebertz A, Rosendaal M, Meghji S (2003) Hypoxia is a major stimulator of osteoclast formation and bone resorption. J Cell Physiol 196: 2-8.

Badylak SF (2005) Regenerative medicine and developmental biology: the role of the extracellular matrix. Anat Rec B New Anat 287: 36-41.

Bahney CS, Hu DP, Taylor AJ, Ferro F, Britz HM, Hallgrimsson B, Johnstone B, Miclau T, Marcucio RS (2014) Stem cell-derived endochondral cartilage stimulates bone healing by tissue transformation. J Bone Miner Res 29: 1269-1282.

Bianco P, Cancedda FD, Riminucci M, Cancedda R (1998) Bone formation via cartilage models: the "borderline" chondrocyte. Matrix Biol 17: 185-192.

Boyan BD, Schwartz Z, Swain LD (1992) In vitro studies on the regulation of endochondral ossification by vitamin D. Crit Rev Oral Biol Med 3: 15-30.

Chen G, Deng C, Li Y-P (2012) TGF- $\beta$ and BMP signaling in osteoblast differentiation and bone formation. Int J Biol Sci 8: 272-288.

Connelly JT, Wilson CG, Levenston ME (2008) Characterization of proteoglycan production and processing by chondrocytes and BMSCs in tissue engineered constructs. Osteoarthritis Cartilage 16: 1092-1100.

Damien CJ, Parsons JR (1991) Bone graft and bone graft substitutes: a review of current technology and applications. J Appl Biomater 2: 187-208.

Demol J, Lambrechts D, Geris L, Schrooten J, Van Oosterwyck H (2011) Towards a quantitative understanding of oxygen tension and cell density evolution in fibrin hydrogels. Biomaterials 32: 107118.

Dimitriou R, Tsiridis E, Giannoudis PV (2005) Current concepts of molecular aspects of bone healing. Injury 36: 1392-1404.

Farrell E, Both SK, Odörfer KI, Koevoet W, Kops N, O'Brien FJ, de Jong RJB, Verhaar JA, Cuijpers V, Jansen J, Erben RG, van Osch GJVM (2011) In-vivo generation of bone via endochondral ossification by in-vitro chondrogenic priming of adult human and rat mesenchymal stem cells. BMC Musculoskelet Disord 12: 31-31.

Gawlitta D, Farrell E, Malda J, Creemers LB, Alblas J, Dhert WJ (2010) Modulating endochondral ossification of multipotent stromal cells for bone regeneration. Tissue Eng Part B Rev 16: 385-395.

Gerber HP, Vu TH, Ryan AM, Kowalski J, Werb Z, Ferrara N (1999) VEGF couples hypertrophic cartilage remodeling, ossification and angiogenesis during endochondral bone formation. Nat Med 5: 623-328.

Hellingman CA, Koevoet W, Kops N, Farrell E, Jahr H, Liu W, Baatenburg de Jong RJ, Frenz DA, van Osch GJ (2010) Fibroblast growth factor receptors in in vitro and in vivo chondrogenesis: relating tissue engineering using adult mesenchymal stem cells to embryonic development. Tissue Eng Part A 16: 545556.

Huang JI, Durbhakula MM, Angele P, Johnstone B, Yoo JU (2006) Lunate arthroplasty with autologous mesenchymal stem cells in a rabbit model. J Bone Joint Surg Am 88: 744-752.

Hung S-P, Ho JH, Shih Y-RV, Lo T, Lee OK (2012) Hypoxia promotes proliferation and osteogenic differentiation potentials of human mesenchymal stem cells. J Orthop Res 30: 260-266.

Jukes JM, Both SK, Leusink A, Sterk LMT, van Blitterswijk CA, de Boer J (2008) Endochondral bone tissue engineering using embryonic stem cells. Proc Natl Acad Sci USA 105: 6840-6845.

Kilkenny C, Browne W, Cuthill IC, Emerson M, Altman DG (2011)Animal research: reporting in vivo experiment-the ARRIVE guidelines. J Cereb Blood Flow Metab 4: 991-993.

Kronenberg HM (2003) Developmental regulation of the growth plate. Nature 423: 332-336.

Lewis MC, MacArthur BD, Tare RS, Oreffo ROC, Please CP (2016) Extracellular matrix deposition in engineered micromass cartilage pellet cultures: measurements and modelling. PLoS One 11: e0147302.

Maes C, Kobayashi T, Selig MK, Torrekens S, Roth SI, Mackem S, Carmeliet G, Kronenberg HM (2010) Osteoblast precursors, but not mature osteoblasts, move into developing and fractured bones along with invading blood vessels. Dev Cell 19: 329-344.

Nagai H, Aoki M (2002) Inhibition of growth plate angiogenesis and endochondral ossification with diminished expression of MMP-13 in hypertrophic chondrocytes in FGF-2-treated rats. J Bone Miner Metab 20: 142-147.

Olsen BR, Reginato AM, Wang W (2000) Bone development. Annu Rev Cell Dev Biol 16: 191-220.

Ortega N, Behonick DJ, Werb Z (2004) Matrix remodeling during endochondral ossification. Trends Cell Biol 14: 86-93.

Osinga R, Di Maggio N, Todorov A, Allafi N, Barbero A, Laurent F, Schaefer DJ, Martin I, Scherberich A (2016) Generation of a bone organ by human adipose-derived stromal cells through endochondral ossification. Stem Cells Transl Med 5: 1090-1097.

Savion N, Vlodavsky I, Fuks Z (1984) Interaction of $\mathrm{T}$ lymphocytes and macrophages with cultured vascular endothelial cells: attachment, invasion, and subsequent degradation of the subendothelial extracellular matrix. J Cell Physiol 118: 169-178.

Scotti C, Piccinini E, Takizawa H, Todorov A, Bourgine P, Papadimitropoulos A, Barbero A, Manz MG, Martin I (2013) Engineering of a functional bone organ through endochondral ossification. Proc Natl Acad Sci USA 110: 3997-4002.

Scotti C, Tonnarelli B, Papadimitropoulos A, Scherberich A, Schaeren S, Schauerte A, Lopez-Rios J, Zeller R, Barbero A, Martin I (2010) Recapitulation of endochondral bone formation using human adult mesenchymal stem cells as a paradigm for developmental engineering. Proc Natl Acad Sci USA 107: 7251-7256.

Sheehy EJ, Vinardell T, Buckley CT, Kelly DJ (2013) Engineering osteochondral constructs through 
spatial regulation of endochondral ossification. Acta Biomater 9: 5484-5492.

Steinbrech DS, Mehrara BJ, Saadeh PB, Chin G, Dudziak ME, Gerrets RP, Gittes GK, Longaker MT (1999) Hypoxia regulates VEGF expression and cellular proliferation by osteoblasts in vitro. Plast Reconstr Surg 104: 738-747.

Street J, Bao M, deGuzman L, Bunting S, Peale FV, Ferrara N, Steinmetz H, Hoeffel J, Cleland JL, Daugherty A, van Bruggen N, Redmond HP, Carano RAD, Filvaroff EH (2002) Vascular endothelial growth factor stimulates bone repair by promoting angiogenesis and bone turnover. Proc Natl Acad Sci USA 99: 9656-9661.

Tortelli F, Tasso R, Loiacono F, Cancedda R (2010) The development of tissue-engineered bone of different origin through endochondral and intramembranous ossification following the implantation of mesenchymal stem cells and osteoblasts in a murine model. Biomaterials 31: 242249.

van der Stok J, Koolen MKE, Jahr H, Kops N, Waarsing JH, Weinans H, van der Jagt OP (2014) Chondrogenically differentiated mesenchymal stromal cell pellets stimulate endochondral bone regeneration in critical-sized bone defects. Eur Cell Mater 19: 137-148.

Yang W, Both SK, van Osch GJ, Wang Y, Jansen JA, Yang F (2015) Effects of in vitro chondrogenic priming time of bone-marrow-derived mesenchymal stromal cells on in vivo endochondral bone formation. Acta Biomater 13: 254-265.

Yang Y-Q, Tan Y-Y, Wong R, Wenden A, Zhang L-K, Rabie ABM (2012) The role of vascular endothelial growth factor in ossification. Int J Oral Sci 4: 64-68.

\section{Discussion with Reviewers}

Reviwer 1: Based on your results, would there be a way to screen donors for their endochondral potential? If so, what criteria for initial chondrogenesis do you believe would be important after in vitro priming to ensure good in vivo results: is it a matrix property, a cellular phenotype or both?

Authors: This is a very interesting and relevant question. At this point, we cannot state what exactly determines ultimate bone formation. We observed a less glycosaminoglycan- and collagenrich matrix resulting in greater bone formation following $28 \mathrm{~d}$ of priming. However, as observed in our $7 \mathrm{~d}$ pellets, there must be a certain quantity of glycosaminoglycan and collagen, or a specific level of cellular differentiation achieved, in order for bone formation to occur. As the cellular phenotype tends to dictate the secreted profile of these constructs, it is most plausible that this is what controls successful bone formation, but what this phenotype is, is not clear yet. It should also be noted that, based on many recent experiments performed in our lab involving subcutaneous implantation, it appears that pellets with a very dense matrix take longer to form bone, likely due to the requirement for more matrix to be broken down and remodelled, but, ultimately, they form bone.

Reviewer 1: Based on the premise of developmental engineering, one would assume that more hypertrophic cartilage, expressing more collagen type $X$ and increased proteoglycan deposition, would be further along the endochondral pathway and therefore convert to bone more quickly. However, this is not supported by the results, given that systems with less hypertrophy and decreased GAG production had better bone formation. Given this, what do the authors suggest promoted endochondral ossification? Did these constructs go through hypertrophic maturation in vivo or are the constructs merely osteoconductive?

Authors: Although it is a common assumption that hypertrophic differentiation dictates bone formation in endochondral constructs, our results showed that there were other factors, outside of this controlling bone formation, involved. Although the extracellular matrix (ECM) is known to play a critical role in bone formation, factors produced by MSCs and the cells themselves have been shown to be crucial for bone formation. The less dense matrix, which allowed for a faster cell invasion and quicker matrix remodelling, in combination with the secreted factors produced by chondrogenically differentiated MSCs, was what we believed contributed to the bone formation we observed. However, given a longer in vivo period, we believe that the pellets with a more GAG-rich, dense and Col X-positive matrix would also have formed bone. Unpublished data from our group would seem to confirm this hypothesis.

Barbero Andrea: The authors speculated that fibrin was able to enhance bone formation due to its ability to reduce oxygen diffusion and tension. Could the authors comment on the possible additional role of fibrin in enhancing the recruitment of inflammatory cells and/or osteoprogenitors, thus allowing for a more efficient remodelling of the cartilage pellets in bone?

Authors: Fibrin can recruit monocytic cells, which have the potential to further differentiate into bone remodelling osteoclasts. This could result in accelerated matrix remodelling. Fibrin also supports the formation of blood vessels and proliferation of mesenchymal stem cells, which would be advantageous for bone formation. In order to determine if these cells were recruited or their activity was enhanced by the presence of fibrin in our study a longitudinal in vivo study would be required.

Barbero Andrea: Which specific bone defects are envisioned to be treated with the described approach? 
Authors: In future studies, we want to optimise these constructs as an injectable bone substitute. As injectable, $\mu$ pellet constructs would be ideal for use in non-critical, irregular or more complex-shaped bone defects, as they would allow for a tailored filling of the void, for example the maxillary sinus, or, potentially, for restoring some structure in cases of avascular necrosis of the femoral head.

Editor note: The scientific editor for this paper was Martin Stoddart. 